\title{
On Economic Equilibrium Type Problems with Applications
}

\author{
Zdzisław Naniewicz
}

Received: 20 June 2010 / Accepted: 13 October 2010 /

Published online: 3 November 2010

(C) The Author(s) 2010. This article is published with open access at Springerlink.com

\begin{abstract}
The existence solution for a class of economic equilibrium type problems in reflexive Banach space is considered. New results concerning the variational inequality approach to Arrow-Debreu model of economic equilibrium introduced in Naniewicz (Math Oper Res 32:436-466, 2007) are found and applied to ensure the existence of Pareto optimal solutions for a class of multiobjective optimization problems with so-called budget-like constraints. To achieve this goal, the theory of pseudo-monotone multivalued mappings combined with some fixed point technique for multivalued mappings with nonconvex values is used.
\end{abstract}

Keywords Economic equilibrium • Constrained multiobjective optimization • Variational inequalities - Pseudo-monotone multivalued operators • Ky Fan inequality $\cdot$ Fixed point

Mathematics Subject Classifications (2010) $49 \mathrm{~J} 40$ • 91B50 • $90 \mathrm{C} 46$

\section{Introduction}

We study the inclusion problem

$$
0 \in \mathcal{R}(x)+\partial f_{0}(x)
$$

for a nonmonotone multivalued mapping $\mathcal{R}: \mathcal{K} \rightarrow 2^{\mathcal{K}^{\star}}$ of the form

$$
\mathcal{R}(x)=\sum_{j=1}^{m} \partial f_{j}\left(\Lambda_{j}(x) x\right), \quad x \in \mathcal{K}
$$

Z. Naniewicz $(\bowtie)$

Faculty of Mathematics and Natural Sciences, College of Sciences,

Cardinal Stefan Wyszyński University, Room 113,

Auditorium Maximum, Wóycickiego 1/3, 01-938, Warsaw, Poland

e-mail: naniewicz.z@acn.waw.pl 
where $\pi \in \mathcal{R}(x)$ iff there exist $\pi_{j} \in X^{\star}, \alpha_{j} \in \mathbb{R}$ such that $\pi=\sum_{j=1}^{m} \pi_{j}, \pi_{j} \in \partial f_{j}\left(\alpha_{j} x\right)$ and $-\left\langle x, \pi_{j}\right\rangle-\phi_{j}(x) \in \partial \operatorname{ind}_{\geq 0}\left(\alpha_{j}\right) . \Lambda_{j}: \mathcal{K} \rightarrow 2^{\mathbb{R}_{+}}$stands for a multivalued mapping given by

$$
\alpha_{j} \in \Lambda_{j}(x)=\left\{\beta_{j} \in \mathbb{R}_{+}: \exists \tau_{j} \in \partial f_{j}\left(\beta_{j} x\right) \text { such that }-\left\langle x, \tau_{j}\right\rangle-\phi_{j}(x) \in \partial \operatorname{ind}_{\geq 0}\left(\beta_{j}\right)\right\}
$$

$j=1, \ldots, m$. Here $\mathcal{K} \subset X$ is a closed convex cone in a reflexive Banach space $X, X^{\star}$ is the dual of $X, \mathcal{K}^{\star} \subset X^{\star}$ is the negative polar cone of $\mathcal{K}$. It is worth pointing out that we do not require the cone $\mathcal{K}$ having nonempty interior.

The following assumptions will be needed throughout the paper: $f_{j}: X \rightarrow$ $\mathbb{R} \cup\{+\infty\}, j=0,1, \ldots, m$, are convex, lower semicontinuous functions such that $\operatorname{Dom}\left(f_{0}\right) \subset \mathcal{K}$ and $\operatorname{Dom}\left(f_{j}^{\star}\right) \subset \mathcal{K}^{\star}, j=1, \ldots, m$, while $\phi_{j}: \mathcal{K} \rightarrow \mathbb{R}_{+}, j=1, \ldots, m$, are continuous, nonnegative valued functions on $\mathcal{K}$. " $\partial$ " stands for the subdifferential operator in the sense of Convex Analysis, ind $\geq_{\geq 0}: \mathbb{R} \rightarrow \mathbb{R} \cup\{+\infty\}$ is the indicator function of the segment $[0,+\infty)$. A symbol "Dom" is used to denote the effective domain of the corresponding function, $\mathbb{R}_{+}=\{t \in \mathbb{R}: t \geq 0\}$.

The problem (1) can be reformulated to the system of subdifferential inclusions

$$
\left.\begin{array}{c}
\pi_{j} \in \partial f_{j}\left(\alpha_{j} x\right), \quad j=1, \ldots, m \\
-\sum_{j=1}^{m} \pi_{j} \in \partial f_{0}(x) \\
\left.\pi_{j}\right\rangle \phi_{j}(x) \in \partial \operatorname{ind}_{\geq 0}\left(\alpha_{j}\right), \quad j=1, \ldots, m
\end{array}\right\}
$$

with the unknowns $x \in \mathcal{K},\left(\pi_{j}\right) \in\left(\mathcal{K}^{\star}\right)^{m},\left(\alpha_{j}\right) \in \mathbb{R}_{+}^{m}$ to be found. In what follows, it will be referred to as the economic equilibrium type problem. Our main result on this issue can be formulated as follows.

Theorem 1 Assume that for each $j=1, \ldots, m$ the hypotheses below hold:

$$
\begin{aligned}
& \left(H_{1}\right) \quad 0 \in \operatorname{cl}\left(\operatorname{Dom}\left(f_{j}^{\star}\right)\right) \text { and } \forall y \in \mathcal{K} \backslash\{0\} \exists \beta \geq 0: \beta y \in \operatorname{Int}\left(\operatorname{Dom}\left(f_{j}\right)\right) \\
& \left(H_{2}^{\prime}\right) \quad \exists \rho_{j}>0, \exists M_{j}>0:\left\{\tau \in \mathcal{K}^{\star}:\left\langle\partial f_{j}^{\star}(\tau) \tau\right\rangle \cap\left(\mathbb{R}_{-}+\rho_{j}\right)=\emptyset\right\} \subset B\left(0, M_{j}\right) \\
& \left(H_{3}^{\prime}\right) \quad \phi_{j}(x) \geq 0 \forall x \in \mathcal{K} \\
& \left(H_{4}\right) \quad \partial f_{j}(0) \text { is locally compact } \\
& \left(H_{5}\right) \quad \phi_{j}: \mathcal{K} \rightarrow \mathbb{R}_{+} \text {is weakly continuous on } \mathcal{K} \\
& \left(H_{6}^{\prime}\right) \quad \exists \rho>0, \exists M>0:\left\{y \in \mathcal{K}: f_{0}(y) \leq \sum_{j=1}^{m} \phi_{j}(y)+f_{0}(0)+\rho\right\} \subset B(0, M) \\
& \left(H_{7}\right) \quad\left(-\sum_{j=1}^{m} \partial f_{j}(0)\right) \cap \partial f_{0}(0)=\emptyset \\
& \left(H_{9}\right) \quad f_{j}^{\infty}(y)+\phi_{j}(y)>0, \forall y \in \mathcal{K} .
\end{aligned}
$$

Then there exist disjoint sets $J, J_{0} \subset\{1, \ldots, m\}$ with $J \cup J_{0}=\{1, \ldots, m\}$ and $x \in \mathcal{K} \backslash 0$, $\left(\pi_{j}\right) \in\left(\mathcal{K}^{\star}\right)^{m},\left(\alpha_{j}\right)_{j \in J} \in \operatorname{Int}\left(\mathbb{R}_{+}\right)^{|J|},\left(\alpha_{j}\right)_{j \in J_{0}}=0 \in\left(\mathbb{R}_{+}\right)^{\left|J_{0}\right|}$, such that

$$
\left.\begin{array}{c}
\pi_{j} \in \partial f_{j}\left(\alpha_{j} x\right), \quad\left\langle x, \pi_{j}\right\rangle+\phi_{j}(x)=0, \quad j \in J \\
\pi_{j} \in \partial f_{j}(0), \quad\left\langle x, \pi_{j}\right\rangle+\phi_{j}(x) \geq 0, \quad j \in J_{0} \\
-\sum_{j=1}^{m} \pi_{j} \in \partial f_{0}(x) .
\end{array}\right\}
$$


Two types of applications of the aforementioned theorem will be presented in this paper:

- first, applications related to economic equilibrium problems,

- second, applications related to a class of multiobjective optimization problems with, what will be called here, "budget-like" constraints.

If $\phi_{j} \geq 0 j=1, \ldots, m$, are convex, positive homogeneous functions and $f_{0}(y)=$ $\sum_{j=1}^{m} \phi_{j}(y), \quad y \in \mathcal{K}$, then (2) can be seen as the variational formulation of the Arrow-Debreu model of economic equilibrium. This formulation has been introduced and primarily studied in [34].

$f_{0}(y)=\sup _{\tau \in \Delta^{\star}}\langle y, \tau\rangle, y \in X, \Delta^{\star} \subset X^{\star}$ being a convex, closed, nonempty set of $\mathcal{K}^{\star}$, characterizes the total budget function while $f_{j}^{\star}$ (the conjugate of $f_{j}$ ) and $\phi_{j}$ are dis-utility and budget functions corresponding to $j$ 's consumer, respectively. $-\pi_{j} \in-\mathcal{K}^{\star}, j=1, \ldots, m$, are the commodity bundles related to $j$ 's consumer and $x \in \mathcal{K}$ represents the price vector.

Indeed, if $\alpha_{j}>0, j=1, \ldots, m$, then (2) ensures $\left(x,\left(\pi_{j}\right)\right) \in \mathcal{K} \times\left(\mathcal{K}^{\star}\right)^{m}$ to be a solution of the problem

$$
\left.\begin{array}{l}
f_{j}^{\star}\left(\pi_{j}\right)=\inf \left\{f_{j}^{\star}(\tau):-\langle x, \tau\rangle \leq \phi_{j}(x) \text { and } \tau \in \mathcal{K}^{\star}\right\}, \quad j=1, \ldots, m \\
f_{0}(x)=-\sum_{j=1}^{m}\left\langle x, \pi_{j}\right\rangle
\end{array}\right\}
$$

i.e., $\left(x,\left(\pi_{j}\right)\right)$ is the Walrasian Equilibrium for the corresponding economic equilibrium problem (cf. [28]). There are numerous literature references related to general equilibrium problem. For this issue we refer the reader to [1-6, 14-16, 19, 22, 24, 2629, 33, 36-39, 45, 46] and the references therein.

Our main result related to the economic equilibrium issues generalizes that of [34] where $X$ has been assumed to be separable. It reads as follows.

Theorem 2 Let $X$ be a reflexive Banach space and $\mathcal{K}^{\star}$ be normal. Moreover, assume that for each $j \in\{1, \ldots, m\}$ the hypotheses below hold:

$$
\begin{array}{ll}
\left(H_{1}^{\prime}\right) & 0 \in \operatorname{cl}\left(\operatorname{Dom}\left(f_{j}^{\star}\right)\right) \\
\left(H_{3}^{\prime \prime \prime}\right) & \exists \gamma>0: \sum_{j=1}^{m} \phi_{j}(y) \geq \gamma\|y\| \quad \forall y \in \mathcal{K} \\
\left(H_{4}\right) & \partial f_{j}(0) \text { is locally compact } \\
\left(H_{7}^{\prime}\right) & \left(-\sum_{j=1}^{m} \partial f_{j}(0)\right) \cap \Delta^{\star}=\emptyset \\
\left(H_{8}^{\prime}\right) & \exists C>0: \Delta^{\star} \cap\left(-\mathcal{K}^{\star}\right) \subset B(0, C) \\
\left(H_{9}^{\prime}\right) & \phi_{j}(y)+f_{j}^{\infty}(y)>0 \forall y \in \mathcal{K} \backslash\{0\} \\
\left(H_{10}^{\prime}\right) & 0 \notin \partial f_{j}(0) .
\end{array}
$$

Then there exist $\left(x,\left(\pi_{j}\right),\left(\alpha_{j}\right)\right) \in \mathcal{K} \times\left(\mathcal{K}^{\star}\right)^{m} \times\left(\mathbb{R}_{+}\right)^{m},\|x\|=1$, and $r \in(0,1]$ such that

$$
\left.\begin{array}{c}
\pi_{j} \in \partial f_{j}\left(\alpha_{j} x\right), \quad j=1, \ldots, m \\
-\sum_{j=1}^{m} \pi_{j} \in r \partial f_{0}(x) \\
-\left\langle x, \pi_{j}\right\rangle-\phi_{j}(x) \in \partial \operatorname{ind}_{\geq 0}\left(\alpha_{j}\right), \quad j=1, \ldots, m .
\end{array}\right\}
$$

Note that $x$ is then a solution of the inclusion

$$
0 \in \mathcal{R}(x)+r \partial f_{0}(x) .
$$


Concerning the second group of applications related to multiobjective optimization issues we replace $f_{j}$ by $f_{j}^{\beta}(y)=f_{j}\left(\frac{1}{\beta_{j}} y\right), y \in X, \beta=\left(\beta_{j}\right) \in \operatorname{Int}\left(\mathbb{R}_{+}^{m}\right)$ and obtain the existence of solution to the problem

$$
\left.\begin{array}{c}
\pi_{j}^{\beta} \in \frac{1}{\beta_{j}} \partial f_{j}\left(\frac{\alpha_{j}^{\beta}}{\beta_{j}} x^{\beta}\right) \\
-\sum_{j=1}^{m} \pi_{j}^{\beta} \in \partial f_{0}\left(x^{\beta}\right) \\
-\left\langle x^{\beta}, \pi_{j}^{\beta}\right\rangle-\phi_{j}\left(x^{\beta}\right)=0
\end{array}\right\} \quad j=1, \ldots, m .
$$

Next, we show that the multivalued mapping $\Gamma(\beta)=\left\{\alpha^{\beta}\right\}$ with nonconvex values admits a fixed point. This leads to the existence of $x^{\beta} \in \mathcal{K}$ such that

$$
\left.\begin{array}{c}
\sum_{j=1}^{m} \frac{1}{\beta_{j}} f_{j}\left(x^{\beta}\right)+f_{0}\left(x^{\beta}\right) \leq \sum_{j=1}^{m} \frac{1}{\beta_{j}} f_{j}(y)+f_{0}(y) \quad \forall y \in \mathcal{K} \\
-\left\langle x^{\beta}, \pi_{j}^{\beta}\right\rangle-\phi_{j}\left(x^{\beta}\right)=0, \quad j=1, \ldots, m .
\end{array}\right\}
$$

Therefore we are allowed to conclude that a multiobjective optimization problem related to the vector objective $\left(f_{0}, f_{1}, \ldots, f_{m}\right)$ possesses at least one Pareto optimal solution fulfilling the "budget-like" constraints $(4)_{2}$.

Our result extends those obtained in [35] and reads as follows.

Theorem 3 Assume that $\mathcal{K}^{\star}$ is normal and $f_{0}(y)=\sum_{j=1}^{m} \phi_{j}(y), \quad y \in \mathcal{K}$, where $\phi_{j}(\cdot)$ are convex, lower semicontinuous, positive homogeneous, nonnegative valued. Suppose that for each $j=1, \ldots$, $m$ the hypotheses below hold:

$\left(H_{1}^{\prime}\right) \quad 0 \in \operatorname{cl}\left(\operatorname{Dom}\left(f_{j}^{\star}\right)\right)$

$\left(H_{3}^{\prime \prime}\right) \quad \exists \gamma_{j}>0: \phi_{j}(y) \geq \gamma_{j}\|y\| \quad \forall y \in \mathcal{K}$

$\left(H_{8}^{\prime}\right) \quad \exists C>0: \Delta^{\star} \cap\left(-\mathcal{K}^{\star}\right) \subset B(0, C)$

$\left(H_{13}\right) \quad \exists G>0: \sum_{j=1}^{m} \phi_{j}(y) \leq G\|y\| \quad \forall y \in \mathcal{K}$.

Moreover, let one of the two assumptions below hold:

$\left(H_{10}^{\prime \prime}\right) \quad \partial f_{j}(0)=\partial f_{j}^{\star}(0)=\emptyset, \quad j=1, \ldots, m$,

or

$\left(H_{12}\right) \quad \lim \sup _{\|y\| \rightarrow \infty} \frac{\left\langle y, \partial f_{j}(y)\right\rangle}{\phi_{j}(y)}<0, \quad j=1, \ldots, m$.

Then there exist $\left(x^{\beta},\left(\pi_{j}^{\beta}\right),\left(\beta_{j}\right)\right)$ such that (4) holds.

There is a vast literature on multiobjective optimization problems. We refer to $[10,11,13,18,20,21,23,25,30-32,40,44,47-50]$ (see also $[1,28]$ ) for a variety approaches, results and discussion.

\section{Preliminaries}

Let $X$ be a reflexive Banach space with its dual $X^{\star}$. The pairing over $X \times X^{\star}$ will be denoted by $\langle\cdot, \cdot\rangle$. Assume $f_{j}: X \rightarrow \mathbb{R} \cup\{+\infty\}, j=0,1, \ldots, m$, to be convex, lower 
semicontinuous, proper functions. Their conjugates in the sense of Convex Analysis will be denoted by $f_{j}^{\star}: X^{\star} \rightarrow \mathbb{R} \cup\{+\infty\}$, respectively. Moreover, assume that there exists a closed, convex cone $\mathcal{K} \subset X$ such that $\operatorname{Dom}\left(f_{0}\right) \subset \mathcal{K}, \operatorname{Dom}\left(f_{j}^{\star}\right) \subset \mathcal{K}^{\star}$, $j=1, \ldots, m$, where $\mathcal{K}^{\star}=\left\{\tau \in X^{\star}:\langle y, \tau\rangle \leq 0 \forall y \in \mathcal{K}\right\}$ is the negative polar cone of $\mathcal{K}$. In the presented approach we do not assume that $\mathcal{K}$ has nonempty interior. Let $\phi_{j}: \mathcal{K} \rightarrow \mathbb{R}_{+}, j=1, \ldots, m$, be continuous functions on $\mathcal{K}$ with nonnegative values.

Fix $x \in \mathcal{K}, x \neq 0$, and consider the collection of minimization problems:

Problem $\left(\bar{P}_{j}^{x}\right) \quad \bar{v}_{j}^{x}:=\inf \left\{f_{j}^{\star}(\tau)+\operatorname{ind}_{\leq \phi_{j}(x)}\left(A_{x} \tau\right): \tau \in X^{\star}\right\}, \quad j=1, \ldots, m$,

where $A_{x}: X^{\star} \rightarrow \mathbb{R}$ is a linear continuous operator given by

$$
A_{x} \tau=-\langle x, \tau\rangle, \quad \tau \in X^{\star}
$$

and ind $\operatorname{s\phi }_{j}(x)$ stands for the indicator of $\left\{t \in \mathbb{R}: t \leq \phi_{j}(x)\right\}$. It is easy to check that the adjoint of $A_{x}$ takes the form $A_{x}^{\star} \alpha=-\alpha x, \alpha \in \mathbb{R}$, and the conjugate of $\operatorname{ind}_{\leq \phi_{j}(x)}$ is given by $\operatorname{ind}_{\leq \phi_{j}(\pi)}^{\star}(\alpha)=\alpha \phi_{j}(x)+\operatorname{ind}_{\mathbb{R}_{+}}(\alpha), \alpha \in \mathbb{R}$. According to the Fenchel duality theory (cf. $[7,8])$ the dual of $\left(\bar{P}_{j}^{x}\right)$ reads

Problem $\left(\underline{P}_{j}^{x}\right) \quad \underline{v}_{j}^{x}:=\inf \left\{f_{j}(\alpha x)+\alpha \phi_{j}(x)+\operatorname{ind}_{\geq 0}(\alpha): \alpha \in \mathbb{R}\right\}, j=1, \ldots, m$.

From the Fenchel duality theory we get the result.

Proposition 1 Suppose that for any $j=1, \ldots, m$ the assumptions below hold:

$\left(H_{1}\right) \quad 0 \in \operatorname{cl}\left(\operatorname{Dom}\left(f_{j}^{\star}\right)\right)$ and $\forall y \in \mathcal{K} \backslash\{0\} \exists \beta \geq 0: \beta y \in \operatorname{Int}\left(\operatorname{Dom}\left(f_{j}\right)\right)$

$\left(H_{2}\right) \quad \exists M_{j}>0:\left\{\tau \in \mathcal{K}^{\star}:\left\langle\partial f_{j}^{\star}(\tau) \tau\right\rangle \cap \mathbb{R}_{-} \neq \emptyset\right\} \subset B\left(0, M_{j}\right)$

$\left(H_{3}\right) \quad \exists \delta_{j}>0: \phi_{j}(x) \geq \delta_{j} \forall x \in \mathcal{K}$.

Then for each $x \in \mathcal{K} \backslash\{0\}$ the optimization problems $\left(\bar{P}_{j}^{x}\right)$ and $\left(\underline{P}_{j}^{x}\right)$ have solutions $\pi_{j}^{x} \in \mathcal{K}^{\star}$ and $\alpha_{j}^{x} \geq 0$, respectively, which are interrelated by

$$
\begin{gathered}
\pi_{j}^{x} \in \partial f_{j}\left(\alpha_{j}^{x} x\right) \Leftrightarrow \alpha_{j}^{x} x \in \partial f_{j}^{\star}\left(\pi_{j}^{x}\right) \\
-\left\langle x, \pi_{j}^{x}\right\rangle-\phi_{j}(x) \in \partial \operatorname{ind}_{\geq 0}\left(\alpha_{j}^{x}\right) \Leftrightarrow \alpha_{j}^{x} \in \partial \operatorname{ind}_{\leq 0}\left(-\left\langle x, \pi_{j}^{x}\right\rangle-\phi_{j}(x)\right) \\
\bar{v}_{j}^{x}+\underline{v}_{j}^{x}=0 .
\end{gathered}
$$

Moreover, if we define $\Lambda_{j}: \mathcal{K} \rightarrow 2^{\mathbb{R}_{+}}$by

$$
\Lambda_{j}(x)= \begin{cases}\left\{\beta_{j} \in \mathbb{R}: \exists \tau_{j} \in \partial f_{j}\left(\beta_{j} x\right) \text { with }-\left\langle x, \tau_{j}\right\rangle-\phi_{j}(x) \in \partial \text { ind }_{\geq 0}\left(\beta_{j}\right)\right\} & x \in \mathcal{K} \backslash 0 \\ 0 & x=0\end{cases}
$$

$j=1, \ldots, m$, then the graph of multivalued mapping $\Lambda_{j}$ is closed and $\Lambda_{j}$ has nonempty, bounded, closed and convex values. 
Proof This is an easy task to check that for $x \in \mathcal{K} \backslash\{0\},\left(H_{1}\right)$ implies

$$
0 \in \operatorname{Int}\left[A_{x} \operatorname{Dom}\left(f_{j}^{\star}\right)-\operatorname{Dom}\left(\operatorname{ind}_{\leq \phi_{j}(x)}\right)\right]
$$

and

$$
0 \in \operatorname{Int}\left[A_{x}^{\star} \operatorname{Dom}\left(\operatorname{ind}_{\leq \phi_{j}(x)}^{\star}\right)+\operatorname{Dom}\left(f_{j}\right)\right]
$$

which due to the theorem of Fenchel [42] (see also [7]) there exist $\pi_{j}^{x} \in \mathcal{K}^{\star}$ and $\alpha_{j}^{x} \geq 0$ such that (6), (7) and (8) hold. Accordingly, we conclude that for any $x \in \mathcal{K} \backslash\{0\}$, $\Lambda_{j}(x)$ is non empty because $\alpha_{j}^{x} \in \Lambda_{j}(x)$.

Furthermore, one can easily check that $\Lambda_{j}(x)$ consists of all solutions of variational inequality

$$
\left\langle x, \partial f_{j}\left(\alpha_{j}^{x} x\right)\right\rangle\left(t-\alpha_{j}^{x}\right)+\phi_{j}(x)\left(t-\alpha_{j}^{x}\right) \geq 0, \quad \forall t \geq 0,
$$

involving maximal monotone mapping $G_{j}^{x}(t):=\left\langle x, \partial f_{j}(t x)\right\rangle, t \geq 0$. Thus, it is a convex and closed set (see [17]).

Now we claim that $\Lambda_{j}(x), x \in \mathcal{K} \backslash\{0\}$ is bounded. To show this let $\alpha_{j}^{x} \in \Lambda_{j}(x)$. From $\alpha_{j}^{x} x \in \partial f_{j}^{\star}\left(\pi_{j}^{x}\right)$ it follows that

$$
0 \geq \alpha_{j}^{x}\left\langle x, \pi_{j}^{x}\right\rangle \in\left\langle\partial f_{j}^{\star}\left(\pi_{j}^{x}\right), \pi_{j}^{x}\right\rangle .
$$

Due to $\left(H_{2}\right)$ this yields $\left\|\pi_{j}^{x}\right\| \leq M_{j}$ for some $M_{j}>0$. This fact, by the lower semicontinuity and convexity of $f_{j}^{\star}$, implies

$$
f_{j}^{\star}\left(\pi_{j}^{x}\right) \geq-a_{j}\left\|\pi_{j}^{x}\right\|-b_{j} \geq-a_{j} M_{j}-b_{j}:=-m_{j}, \quad \text { for some } a_{j}, b_{j} \geq 0 .
$$

Since $-\left\langle x, \pi_{j}^{x}\right\rangle-\phi_{j}(x) \in \partial$ ind $_{\geq 0}\left(\alpha_{j}^{x}\right)$, we are led to

$$
-\alpha_{j}^{x}\left\langle x, \pi_{j}^{x}\right\rangle=\alpha_{j}^{x} \phi_{j}(x)
$$

which in view of $\alpha_{j}^{x} x \in \partial f_{j}^{\star}\left(\pi_{j}^{x}\right)$ implies

$$
\alpha_{j}^{x} \phi_{j}(x) \leq m_{j}+f_{j}^{\star}(\tau)-\alpha_{j}^{x}\langle x, \tau\rangle, \quad \forall \tau \in \operatorname{Dom}\left(f_{j}^{\star}\right) .
$$

Now, on the contrary, suppose that $\Lambda_{j}(x)$ is unbounded. There exists a sequence $\left\{\alpha_{j}^{n}\right\} \subset \Lambda_{j}(x)$ with $\alpha_{j}^{n} \rightarrow \infty$ as $n \rightarrow \infty$ and such that

$$
\phi_{j}(x) \leq \frac{m_{j}}{\alpha_{j}^{n}}+\frac{1}{\alpha_{j}^{n}} f_{j}^{\star}(\tau)+\|\tau\|\|x\|, \quad \forall \tau \in \operatorname{Dom}\left(f_{j}^{\star}\right) .
$$

Letting $n \rightarrow \infty$ yields

$$
\phi_{j}(x) \leq\|\tau\|\|x\|, \quad \forall \tau \in \operatorname{Dom}\left(f_{j}^{\star}\right) .
$$

Since 0 is a cluster point of $\operatorname{Dom}\left(f_{j}^{\star}\right)\left(\right.$ due to $\left.\left(H_{1}\right)\right)$, one obtains $\phi_{j}(x) \leq 0$, contrary to $\left(H_{3}\right)$. The boundedness of $\Lambda_{j}(x)$ has been established. 
Now let us notice that for sufficiently small $\epsilon>0$ the condition $\|x\| \leq \epsilon$ implies $-\left\langle x, \pi_{j}^{x}\right\rangle \leq\|x\|\left\|\pi_{j}^{x}\right\| \leq \epsilon M_{j}<\delta_{j} \leq \phi_{j}(x)$. Thus by (11) we deduce that $\alpha_{j}^{x}=0$. Therefore in some neighborhood of 0 in $\mathcal{K}$, say $O(0)$, we have $\Lambda_{j}(x)=\{0\}$ for any $x \in O(0)$, which means that $\Lambda_{j}: \mathcal{K} \rightarrow 2^{\mathbb{R}_{+}}$is continuous at $0 \in \mathcal{K}$.

In order to establish the closedness of the graph of $\Lambda_{j}(\cdot)$ on $\mathcal{K} \times \mathbb{R}_{+}$it remains to check whether from the assumptions: $\left\{x^{n}\right\} \subset \mathcal{K}, \alpha_{j}^{n} \in \Lambda_{j}\left(x^{n}\right), x^{n} \rightarrow x^{\star}$ in $X, \alpha_{j}^{n} \rightarrow \alpha_{j}^{\star}$ in $\mathbb{R}$ for some $x^{\star} \in \mathcal{K}, x^{\star} \neq 0$, and $\alpha_{j}^{\star} \in \mathbb{R}_{+}$, respectively, one can deduce that $\alpha_{j}^{\star} \in$ $\Lambda_{j}\left(x^{\star}\right)$.

Since there exists $\pi_{j}^{n} \in \mathcal{K}^{\star}$ with $\alpha_{j}^{n} x^{n} \in \partial f_{j}^{\star}\left(\pi_{j}^{n}\right)$, we get

$$
\alpha_{j}^{n}\left\langle x^{n}, \pi_{j}^{n}\right\rangle \in\left\langle\partial f_{j}^{\star}\left(\pi_{j}^{n}\right), \pi_{j}^{n}\right\rangle
$$

But the left hand side of this inclusion is non-positive. Therefore, by $\left(H_{2}\right),\left\{\pi_{j}^{n}\right\}$ is bounded. Consequently, one can suppose that $\pi_{j}^{n} \rightarrow \pi_{j}^{\star}$ weakly in $X^{\star}$ for some $\pi_{j}^{\star} \in$ $\mathcal{K}^{\star}$ (by passing to a subsequence, if necessary). Taking into account the conditions

$$
\begin{gathered}
\alpha_{j}^{n} x^{n} \in \partial f_{j}^{\star}\left(\pi_{j}^{n}\right) \\
-\left\langle x^{n}, \pi_{j}^{n}\right\rangle-\phi_{j}\left(x^{n}\right) \in \partial \operatorname{ind}_{\geq 0}\left(\alpha_{j}^{n}\right),
\end{gathered}
$$

we are allowed to pass to the limit as $n \rightarrow \infty$. By the continuity of $\phi_{j}(\cdot)$, the maximal monotonicity of $\partial f_{j}^{\star}(\cdot)$ and $\partial \operatorname{ind}_{\geq 0}(\cdot)$, we get

$$
\begin{gathered}
-\alpha_{j}^{\star} x^{\star} \in \partial f_{j}^{\star}\left(\pi_{j}^{\star}\right) \\
-\left\langle x^{\star}, \pi_{j}^{\star}\right\rangle-\phi_{j}\left(x^{\star}\right) \in \partial \operatorname{ind}_{\geq 0}\left(\alpha_{j}^{\star}\right)
\end{gathered}
$$

from which it follows $\alpha_{j}^{\star} \in \Lambda_{j}\left(x^{\star}\right)$, as desired. The proof is complete.

Now we introduce a multivalued mapping $\mathcal{R}: \mathcal{K} \rightarrow 2^{\mathcal{K}^{\star}}$ by setting

$$
\begin{aligned}
\mathcal{R}(x)= & \sum_{j=1}^{m} \partial f_{j}\left(\Lambda_{j}(x) x\right) \\
= & \left\{\pi \in X^{\star}: \exists \alpha_{j} \in \mathbb{R}_{+}, \exists \pi_{j} \in \partial f_{j}\left(\alpha_{j} x\right) \text { with } \pi=\sum_{j=1}^{m} \pi_{j}\right. \text { and } \\
& \left.\quad-\left\langle x, \pi_{j}\right\rangle-\phi_{j}(x) \in \partial \operatorname{ind}_{\geq 0}\left(\alpha_{j}\right), j=1, \ldots, m\right\}, \quad x \in \mathcal{K} .
\end{aligned}
$$

Note that $\mathcal{R}(\cdot)$ is not monotone due to the dependence of $\Lambda_{j}$ on $x$.

Proposition 2 Under the assumptions of Proposition 1, $\mathcal{R}$ given by (13) is a uniformly bounded multivalued mapping with nonempty, convex, closed values.

Proof To begin with let us notice that if $\tau \in \mathcal{R}(y), \tau \in \mathcal{K}$, then $\tau=\sum_{j=1}^{m} \tau_{j}$ with $\tau_{j} \in \partial f_{j}\left(\beta_{j} y\right)$ and $\beta_{j} \in \Lambda_{j}(y)$. Hence $\left\langle\partial f_{j}^{\star}\left(\tau_{j}\right), \tau_{j}\right\rangle \ni \beta_{j}\left\langle y, \tau_{j}\right\rangle \leq 0$ which by $\left(H_{2}\right)$ implies $\left\|\tau_{j}\right\| \leq M_{j}$. Consequently, $\|\tau\| \leq \sum_{j=1}^{m} M_{j}$. The boundedness of $\mathcal{R}$ follows. 
Next we show that $\partial f_{j}\left(\alpha_{1} x\right)=\partial f_{j}\left(\alpha_{2} x\right)$ for any $\alpha_{1}, \alpha_{2} \in \Lambda_{j}(x), x \in \mathcal{K}$. To this end, assume that $\tilde{\pi}_{1}, \tilde{\pi}_{2} \in \partial f_{j}\left(\Lambda_{j}(x) x\right)$. There exist $\alpha_{1}, \alpha_{2} \in \Lambda_{j}(x)$ with the property that

$$
\tilde{\pi}_{1} \in \partial f_{j}\left(\alpha_{1} x\right) \text { and } \tilde{\pi}_{2} \in \partial f_{j}\left(\alpha_{2} x\right) .
$$

For $t \in[0,1]$ set $\tilde{\pi}_{t}=t \tilde{\pi}_{1}+(1-t) \tilde{\pi}_{2}$. Since $\tilde{\pi}_{1}$ and $\tilde{\pi}_{2}$ are solutions of $\left(\bar{P}_{j}^{x}\right)$, we have

$$
\begin{gathered}
f_{j}^{\star}\left(\tilde{\pi}_{1}\right)=f_{j}^{\star}\left(\tilde{\pi}_{2}\right)=f_{j}^{\star}\left(\tilde{\pi}_{t}\right), \\
f_{j}^{\star}\left(\tilde{\pi}_{n}\right)+f_{j}\left(\alpha_{n} x\right)=\alpha_{n}\left\langle x, \tilde{\pi}_{n}\right\rangle, \\
\alpha_{n}\left(\left\langle x, \tilde{\pi}_{n}\right\rangle+\phi_{j}(x)\right)=0, \quad n=1,2 .
\end{gathered}
$$

Case $1 \alpha_{1}, \alpha_{2}>0$.

Taking into account that $\alpha_{n} x \in \partial f_{j}^{\star}\left(\tilde{\pi}_{n}\right), n=1,2$, we obtain

$$
-\left\langle\alpha_{n} x, \tau-\tilde{\pi}_{n}\right\rangle+f_{j}^{\star}(\tau)-f_{j}^{\star}\left(\tilde{\pi}_{n}\right) \geq 0, \quad \forall \tau \in \mathcal{K}^{\star}, n=1,2 .
$$

Since in such a case $\left\langle x, \tilde{\pi}_{n}\right\rangle+\phi_{j}(x)=0, n=1,2$, we conclude that $\left\langle x, \tilde{\pi}_{t}\right\rangle+\phi_{j}(x)=0$, and consequently

$$
-\left\langle\alpha_{n} x, \tau-\tilde{\pi}_{t}\right\rangle+f_{j}^{\star}(\tau)-f_{j}^{\star}\left(\tilde{\pi}_{t}\right) \geq 0, \quad \forall \tau \in \mathcal{K}^{\star}, n=1,2 .
$$

Hence, by adding these inequalities multiplied by $t^{\star}$ and $1-t^{\star}, t^{\star} \in[0,1]$, respectively, we easily arrive at

$$
-\left\langle\alpha_{t^{\star}} x, \tau-\tilde{\pi}_{t}\right\rangle+f_{j}^{\star}(\tau)-f_{j}^{\star}\left(\tilde{\pi}_{t}\right) \geq 0, \quad \forall \tau \in \mathcal{K}^{\star},
$$

where $\alpha_{t^{\star}}:=t^{\star} \alpha_{1}+\left(1-t^{\star}\right) \alpha_{2}$. Hence $\alpha_{t^{\star}} x \in \partial f_{j}^{\star}\left(\tilde{\pi}_{t}\right)$ for any $t, t^{\star} \in[0,1]$ which is equivalent to $\tilde{\pi}_{t} \in \partial f_{j}\left(\alpha_{t^{\star}} x\right)$. But this means that $\partial f_{j}(\alpha x)$ does not depend on the choice of $\alpha \in \Lambda_{j}(x)$, as claimed.

Case $2 \alpha_{1}=0, \alpha_{2}>0$.

In this case we have

$$
\tilde{\pi}_{1} \in \partial f_{j}(0) \text { and } \quad \tilde{\pi}_{2} \in \partial f_{j}\left(\alpha_{2} x\right)
$$

and

$$
-\left\langle x, \tilde{\pi}_{1}\right\rangle-\phi_{j}(x) \leq 0, \quad\left\langle x, \tilde{\pi}_{2}\right\rangle+\phi_{j}(x)=0 .
$$

From the monotonicity of $\partial f_{j}(\cdot)$ it follows

$$
\left\langle\alpha_{2} x, \tilde{\pi}_{2}-\tilde{\pi}_{1}\right\rangle \geq 0 \text {. }
$$

Hence

$$
-\alpha_{2} \phi_{j}(x)=\left\langle\alpha_{2} x, \tilde{\pi}_{2}\right\rangle \geq\left\langle\alpha_{2} x, \tilde{\pi}_{1}\right\rangle
$$

and consequently, $-\left\langle x, \tilde{\pi}_{1}\right\rangle=\phi_{j}(x)$. Thus we have arrived at the case considered previously. The assertion follows.

To complete the proof it remains to invoke the well known properties of maximal monotone mappings $\partial f_{j}(\cdot)$ and combine them with the properties of $\Lambda_{j}(\cdot)$ mentioned in Proposition 1. 


\section{Pseudo-Monotonicity of $\mathcal{R}$}

Let us recall some definitions and properties of the theory of monotone type mappings.

Definition 1 [12] An operator $T: \mathcal{K} \rightarrow 2^{X^{\star}}$ is said to be pseudo-monotone from $\mathcal{K}$ into $2^{X^{\star}}$ if the following conditions hold:

(1) For any $x \in \mathcal{K}, T(x)$ is nonempty, bounded, closed and convex;

(2) $T$ is upper semicontinuous from each $F \cap \mathcal{K}$ ( $F$ - finite dimensional subspace of $X)$ to the weak topology of $X^{\star}$;

(3) For any $\left\{x^{n}\right\} \subset \mathcal{K}$, the conditions $x^{n} \rightarrow x$ in $X$ and

$$
\limsup _{n \rightarrow \infty}\left\langle x^{n}-x, \tau^{n}\right\rangle \leq 0
$$

with $\tau^{n} \in T\left(x^{n}\right)$ imply that for any $y \in \mathcal{K}$ there exists $\tau(y) \in T(x)$ such that

$$
\liminf _{n \rightarrow \infty}\left\langle x^{n}-y, \tau^{n}\right\rangle \geq\langle x-y, \tau(y)\rangle .
$$

Now we are ready to discuss the pseudo-monotone properties of $\mathcal{R}$.

Proposition 3 Suppose that for any $j \in\{1, \ldots, m\}$ the following conditions hold:

$\left(H_{1}\right) \quad 0 \in \operatorname{cl}\left(\operatorname{Dom}\left(f_{j}^{\star}\right)\right)$ and $\forall y \in \mathcal{K} \backslash\{0\} \exists \beta \geq 0: \beta y \in \operatorname{Int}\left(\operatorname{Dom}\left(f_{j}\right)\right)$

$\left(H_{2}\right) \quad \exists M_{j}>0:\left\{\tau \in \mathcal{K}^{\star}:\left\langle\partial f_{j}^{\star}(\tau), \tau\right\rangle \cap \mathbb{R}_{-} \neq \emptyset\right\} \subset B\left(0, M_{j}\right)$

$\left(H_{3}\right) \quad \exists \delta_{j}>0: \phi_{j}(x) \geq \delta_{j} \forall x \in \mathcal{K}$

$\left(H_{4}\right) \quad \partial f_{j}(0)$ is locally compact

$\left(H_{5}\right) \quad \phi_{j}: \mathcal{K} \rightarrow \mathbb{R}_{+}$is weakly continuous on $\mathcal{K}$.

Then $\mathcal{R}: \mathcal{K} \rightarrow 2^{X^{\star}}$ is a pseudo-monotone mapping on $\mathcal{K}$.

Proof In order to show that $\mathcal{R}$ is pseudo-monotone on $\mathcal{K}$ we have to check that (1)-(3) of Definition 1 hold.

(1) See Proposition 2.

(3) Suppose that $x^{n} \rightarrow x$ weakly in $X,\left\{x^{n}\right\} \subset \mathcal{K}, \pi^{n} \in \mathcal{R}\left(x^{n}\right)$ and

$$
\limsup _{n \rightarrow \infty}\left\langle x^{n}-x, \pi^{n}\right\rangle \leq 0 \text {. }
$$

Our task is to show that for each $y \in \mathcal{K}$ there exists an element $\pi(y) \in X^{\star}$ such that

$$
\begin{gathered}
\pi(y) \in \mathcal{R}(x) \\
\liminf _{n \rightarrow \infty}\left\langle x^{n}-y, \pi^{n}\right\rangle \geq\langle x-y, \pi(y)\rangle .
\end{gathered}
$$

Since $\pi^{n} \in \mathcal{R}\left(x^{n}\right), \pi^{n}$ admits the representation

$$
\pi^{n}=\sum_{j=1}^{m} \pi_{j}^{n},
$$


for some

$$
\pi_{j}^{n} \in \partial f_{j}\left(\alpha_{j}^{n} x^{n}\right)
$$

(or equivalently: $\alpha_{j}^{n} x^{n} \in \partial f_{j}^{\star}\left(\pi_{j}^{n}\right)$ ), where $\alpha_{j}^{n} \in \Lambda_{j}\left(x^{n}\right)$. By the definition of $\Lambda_{j}$,

$$
-\left\langle x^{n}, \pi_{j}^{n}\right\rangle-\phi_{j}\left(x^{n}\right) \in \partial \operatorname{ind}_{\geq 0}\left(\alpha_{j}^{n}\right) .
$$

As it has been already shown, $\left(H_{2}\right)$ implies the boundedness of $\left\{\pi_{j}^{n}\right\}_{n=1}^{\infty} \subset \mathcal{K}^{\star}$, namely $\left\|\pi_{j}^{n}\right\| \leq M_{j}, j=1, \ldots, m$. For the boundedness of $\left\{\alpha_{j}^{n}\right\} \subset \mathbb{R}_{+}$we proceed like in the proof of Proposition 1. Namely, analogously to (12) it follows

$$
\alpha_{j}^{n} \phi_{j}\left(x^{n}\right) \leq m_{j}+f_{j}^{\star}(\tau)-\alpha_{j}^{n}\left\langle x^{n}, \tau\right\rangle, \quad \forall \tau \in \operatorname{Dom}\left(f_{j}^{\star}\right) .
$$

Hence we get

$$
\phi_{j}\left(x^{n}\right) \leq \frac{m_{j}}{\alpha_{j}^{n}}+\frac{1}{\alpha_{j}^{n}} f_{j}^{\star}(\tau)+\|\tau\|\left\|x^{n}\right\|, \quad \forall \tau \in \operatorname{Dom}\left(f_{j}^{\star}\right) .
$$

If $\alpha_{j}^{n} \rightarrow \infty$ then we are led to the contradiction. Indeed, by using the weak continuity of $\phi_{j}$ and boundedness of $\left\{\|x\|^{n}\right\},\left\|x^{n}\right\| \leq C$, it follows

$$
\phi_{j}(x) \leq C\|\tau\|, \quad \forall \tau \in \operatorname{Dom}\left(f_{j}^{\star}\right) .
$$

By $\left(H_{1}\right), 0$ is a cluster point of $\operatorname{Dom}\left(f_{j}^{\star}\right)$ which implies $\phi_{j}(x) \leq 0$, contrary to $\left(H_{3}\right)$. The assertion follows.

Therefore, without loss of generality it can be assumed that

$$
\begin{gathered}
\pi_{j}^{n} \rightarrow \pi_{j} \quad \text { weakly in } X^{\star} \text { as } n \rightarrow \infty \\
\alpha_{j}^{n} \rightarrow \alpha_{j} \quad \text { in } \mathbb{R} \text { as } n \rightarrow \infty
\end{gathered}
$$

for some $\pi_{j} \in \mathcal{K}^{\star}$ and $\alpha_{j} \in \mathbb{R}_{+}$. From (14) it follows

$$
\limsup _{n \rightarrow \infty}\left\langle x^{n}-x, \sum_{j=1}^{m} \pi_{j}^{n}\right\rangle \leq 0 .
$$

On the other hand, by virtue of (18),

$$
f_{j}\left(\alpha_{j} x\right)-f_{j}\left(\alpha_{j}^{n} x^{n}\right) \geq\left\langle\alpha_{j} x-\alpha_{j}^{n} x^{n}, \pi_{j}^{n}\right\rangle .
$$

Since $\alpha_{j}^{n} x^{n} \rightarrow \alpha_{j} x$ weakly in $X$, the weak lower semicontinuity of $f_{j}$ yields

$$
\liminf _{n \rightarrow \infty}\left\langle\alpha_{j}^{n} x^{n}, \pi_{j}^{n}\right\rangle \geq\left\langle\alpha_{j} x, \pi_{j}\right\rangle .
$$

Now we claim that for any $j \in\{1, \ldots, m\}$,

$$
\liminf _{n \rightarrow \infty}\left\langle x^{n}, \pi_{j}^{n}\right\rangle \geq\left\langle x, \pi_{j}\right\rangle .
$$

Indeed, if $\alpha_{j}>0$, the assertion can be deduced directly from (24). If $\alpha_{j}=0$ then $\alpha_{j}^{n} x^{n} \rightarrow 0$ strongly in $X$. Hence, by the maximal monotonicity of $\partial f_{j}, \pi_{j} \in \partial f_{j}(0)$. Since 
$\partial f_{j}$ is monotone, we have $\left\langle\alpha_{j}^{n} x^{n}, \pi_{j}^{n}-\pi_{j}\right\rangle \geq 0$. It immediately implies (25) provided that $\alpha_{j}^{n}>0$ (for infinitely many $n$ ). If $\alpha_{j}^{n}=0$ for any $n$ then $\pi_{j}^{n} \in \partial f_{j}(0)$ which by $\left(H_{4}\right)$ implies that $\pi_{j}^{n} \rightarrow \pi_{j}$ strongly in $X^{\star}$. Hence $\left\langle x^{n}, \pi_{j}^{n}\right\rangle \rightarrow\left\langle x, \pi_{j}\right\rangle$ as $n \rightarrow \infty$ and (25) follows.

Now combining (25) and (23) yields

$$
\lim _{n \rightarrow \infty}\left\langle x^{n}, \pi_{j}^{n}\right\rangle=\left\langle x, \pi_{j}\right\rangle, \quad \forall j=1, \ldots, m .
$$

Consequently,

$$
\lim _{n \rightarrow \infty}\left\langle x^{n}-y, \pi^{n}\right\rangle=\langle x-y, \pi\rangle
$$

for every $y \in X$, where $\pi=\sum_{j=1}^{m} \pi_{j}$, i.e. (16) holds with $\pi(y):=\pi$.

Now we assert that $\pi \in \mathcal{R}(x)$, or equivalently,

$$
\begin{gathered}
\alpha_{j} x \in \partial f_{j}^{\star}\left(\pi_{j}\right), \quad \forall j=1, \ldots, m \\
-\left\langle x, \pi_{j}\right\rangle-\phi_{j}(x) \in \partial \operatorname{ind}_{\geq 0}\left(\alpha_{j}\right), \quad \forall j=1, \ldots, m .
\end{gathered}
$$

For (27) we need (18). Namely, taking into account that $\alpha_{j}^{n} x^{n} \rightarrow \alpha_{j} x$ and $\pi_{j}^{n} \rightarrow \pi_{j}$ weakly in $X$ and $X^{\star}$, respectively, by (26) we get

$$
\limsup _{n \rightarrow \infty}\left\langle\alpha_{j}^{n} x^{n}-\alpha_{j} x, \pi_{j}^{n}\right\rangle \leq 0 .
$$

Hence the maximal monotonicity of $\partial f_{j}^{\star}$ yields (27).

In order to complete the proof we have to show (28). This in view of (26) and $\left(H_{5}\right)$ can be easily deduced by passing to the limit as $n \rightarrow \infty$ in (19). The proof of condition (2) in the definition of pseudo-monotonicity is complete.

(2) Suppose that $x^{n} \rightarrow x$ in $F \cap \mathcal{K}, F$ being the finite dimensional subspace of $X$, $\pi^{n} \in \mathcal{R}\left(x^{n}\right)$. Since $\mathcal{R}$ is uniformly bounded, $\left\{\pi^{n}\right\}$ is bounded and therefore (14) is fulfilled. As in (3) we show that every weak limit of $\left\{\pi^{n}\right\}$ lies in $\mathcal{R}(x)$. This ensures the desired upper semicontinuity.

The proof of Proposition 3 is complete.

\section{Existence Results}

Consider the problem of finding $x \in \mathcal{K}$ such that for a certain $\pi \in \mathcal{R}(x)$ the following variational inequality holds:

$$
\langle y-x, \pi\rangle+f_{0}(y)-f_{0}(x) \geq 0, \quad \forall y \in \mathcal{K} .
$$

Recall that Dom $f_{0} \subset \mathcal{K}$. Thus this problem can be written equivalently as

$$
0 \in \mathcal{R}(x)+\partial f_{0}(x)
$$

The important point to note here is the fact that both $\mathcal{K}$ and $\mathcal{K}^{\star}$ are allowed to have empty interiors. The reason is that we are going to use the theory of generalized pseudo-monotone mappings developed by Browder-Hess in [12, pp. 288-293]. In this theory the corresponding cone having internal points is not required. 
Proposition 4 Suppose that for any $j=1, \ldots, m$ the assumptions below are fulfilled:

$\left(H_{1}\right) \quad 0 \in \operatorname{cl}\left(\operatorname{Dom}\left(f_{j}^{\star}\right)\right)$ and $\forall y \in \mathcal{K} \backslash\{0\} \exists \beta \geq 0: \beta y \in \operatorname{Int}\left(\operatorname{Dom}\left(f_{j}\right)\right)$

$\left(H_{2}\right) \quad \exists M_{j}:\left\{\tau \in \mathcal{K}^{\star}:\left\langle\partial f_{j}^{\star}(\tau), \tau\right\rangle \cap \mathbb{R}_{-} \neq \emptyset\right\} \subset B\left(0, M_{j}\right)$

$\left(H_{3}\right) \quad \exists \delta_{j}>0: \phi_{j}(x) \geq \delta_{j} \forall x \in \mathcal{K}$

$\left(H_{4}\right) \quad \partial f_{j}(0)$ is locally compact

$\left(H_{5}\right) \quad \phi_{j}: \mathcal{K} \rightarrow \mathbb{R}_{+}$is weakly continuous on $\mathcal{K}$

$\left(H_{6}\right) \quad \exists y_{0} \in \operatorname{Dom}\left(f_{0}\right), \exists M>0:\left\{y \in \mathcal{K}: f_{0}(y) \leq \sum_{j=1}^{m} \phi_{j}(y)+f_{0}\left(y_{0}\right)\right\} \subset B(0, M)$.

Then the problem: Find $x \in \mathcal{K}$ such that

$$
0 \in \partial f_{0}(x)+\mathcal{R}(x)
$$

admits at least one solution. Equivalently, there exists $\left(x,\left(\pi_{j}\right),\left(\alpha_{j}\right)\right) \in \mathcal{K} \times\left(\mathcal{K}^{\star}\right)^{m} \times$ $\left(\mathbb{R}_{+}\right)^{m}$ such that

$$
\left.\begin{array}{c}
\pi_{j} \in \partial f_{j}\left(\alpha_{j} x\right) \\
-\sum_{j=1}^{m} \pi_{j} \in \partial f_{0}(x) \\
-\left\langle x, \pi_{j}\right\rangle-\phi_{j}(x) \in \partial \operatorname{ind}_{\geq 0}\left(\alpha_{j}\right) .
\end{array}\right\}
$$

Proof Introduce the duality mapping $J: X \rightarrow 2^{X^{\star}}$ by $J(y)=\left\{\tau \in X^{\star}:\langle y, \tau\rangle=\right.$ $\|\tau\|\|y\|,\|y\|=\|\tau\|\}$ (cf. [12], p. 280). Recall that $J(\cdot)=\partial\left(\frac{1}{2}\|\cdot\|^{2}\right)$.

For a maximal monotone mapping $T: X \rightarrow 2^{X^{\star}}$ define its Yosida approximation $T_{\lambda}: X \rightarrow 2^{X^{\star}}, \lambda>0$, by $T_{\lambda}:=\left(T^{-1}+\lambda J^{-1}\right)^{-1}$. It is well known that for any $\lambda>0$, $T_{\lambda}$ is a maximal monotone, bounded operator with Dom $T_{\lambda}=X$. Thus it is pseudomonotone on $X$ (cf. [12], Proposition 8, p. 266).

Now, let us set $T=\partial f_{0}$ and $T_{\lambda}=\left(\partial f_{0}\right)_{\lambda}$. Proceeding like in the proof of ([12], Proposition 9 , p. 267) we ensure that $\mathcal{R}+\left(\partial f_{0}\right)_{\lambda}$ is a pseudo-monotone mapping on $\mathcal{K}$ for any $\lambda>0$. Thus applying ([12], Theorem 15, p. 289) we deduce the existence of $x^{\lambda} \in B(0,2 M) \cap \mathcal{K}$ such that

$$
\left\langle y-x^{\lambda}, \mathcal{R}\left(x^{\lambda}\right)+\left(\partial f_{0}\right)_{\lambda}\left(x^{\lambda}\right)\right| \geq 0, \quad \forall y \in \mathcal{K} \cap B(0,2 M),
$$

with $M$ as in $\left(H_{6}\right)$. Thus there exist $\pi_{j}^{\lambda} \in \mathcal{K}^{\star} \cap B\left(0, M_{j}\right), \tau^{\lambda} \in\left(\partial f_{0}\right)_{\lambda}\left(x^{\lambda}\right)$ and $z^{\lambda} \in X$ with the properties that

$$
\sum_{j=1}^{m} \pi_{j}^{\lambda} \in \mathcal{R}\left(x^{\lambda}\right), \quad \tau^{\lambda} \in \partial f_{0}\left(z^{\lambda}\right), \quad \lambda \tau^{\lambda} \in J\left(x^{\lambda}-z^{\lambda}\right)
$$

and

$$
\left\langle y-x^{\lambda}, \sum_{j=1}^{m} \pi_{j}^{\lambda}+\tau^{\lambda}\right\rangle \geq 0, \quad \forall y \in \mathcal{K} \cap B(0,2 M) .
$$

By the definition of $J$ it follows

$$
\left\langle x^{\lambda}-z^{\lambda}, \lambda \tau^{\lambda}\right\rangle=\left\|x^{\lambda}-z^{\lambda}\right\|\left\|\lambda \tau^{\lambda}\right\|, \quad\left\|\lambda \tau^{\lambda}\right\|=\left\|x^{\lambda}-z^{\lambda}\right\| .
$$

Now, substituting $y=y_{0}$ into (35) leads to the estimate

$$
-\left\langle x^{\lambda}, \sum_{j=1}^{m} \pi_{j}^{\lambda}\right\rangle \geq\left\langle x^{\lambda}-z^{\lambda}, \tau^{\lambda}\right\rangle+\left\langle z^{\lambda}-y_{0}, \tau^{\lambda}\right\rangle,
$$


from which we get

$$
\sum_{j=1}^{m} \phi_{j}\left(x^{\lambda}\right) \geq \frac{\left\|x^{\lambda}-z^{\lambda}\right\|^{2}}{\lambda}+f_{0}\left(z^{\lambda}\right)-f_{0}\left(y_{0}\right) .
$$

Taking into account that $f_{0}\left(z^{\lambda}\right) \geq-a\left\|z^{\lambda}\right\|-b$ for some $a, b>0$ ( $f_{0}$ is proper, lower semicontinuous convex) and that $x^{\lambda} \in B(0,2 M)$, one gets

$$
C \geq \sup _{\|y\| \leq 2 M}\left\{\sum_{j=1}^{m} \phi_{j}(y)\right\}+f_{0}\left(y_{0}\right)+2 a M \geq \frac{\left\|x^{\lambda}-z^{\lambda}\right\|^{2}}{\lambda}-a\left\|x^{\lambda}-z^{\lambda}\right\|
$$

for some constant $C>0$. Thus $\left\|x^{\lambda}-z^{\lambda}\right\| \rightarrow 0$ as $\lambda \rightarrow 0$. Since $\left\{x^{\lambda}\right\} \subset B(0,2 M) \cap \mathcal{K}$ and $\mathcal{R}$ is uniformly bounded, one can extract a sequence $\lambda_{n} \rightarrow 0$ such that

$$
\begin{aligned}
& x^{\lambda_{n}} \rightarrow x^{\star}, \quad \text { weakly in } X \text { as } n \rightarrow \infty, \\
& \pi_{j}^{\lambda_{n}} \rightarrow \pi_{j}^{\star} \quad \text { weakly in } X^{\star} \text { as } n \rightarrow \infty,
\end{aligned}
$$

Notice that $z^{\lambda_{n}} \rightarrow x^{\star}$ as well. By substituting $y=x^{\star}$ into (35) we obtain

$$
f_{0}\left(x^{\star}\right)-f_{0}\left(z^{\lambda_{n}}\right)-\frac{\left\|x^{\lambda_{n}}-z^{\lambda_{n}}\right\|^{2}}{\lambda_{n}} \geq\left\langle x^{\lambda_{n}}-x^{\star}, \sum_{j=1}^{m} \pi_{j}^{\lambda_{n}}\right\rangle
$$

from which, by the weak lower semicontinuity of $f_{0}$, it follows

$$
0 \geq \limsup _{n \rightarrow \infty}\left[\left\langle x^{\lambda_{n}}-x^{\star}, \sum_{j=1}^{m} \pi_{j}^{\lambda_{n}}\right\rangle\right]
$$

Now applying the pseudo-monotonicity of $\mathcal{R}$ yields

$$
\sum_{j=1}^{m} \pi_{j}^{\star} \in \mathcal{R}\left(x^{\star}\right), \quad \lim _{n \rightarrow \infty}\left\langle x^{\lambda_{n}}-x^{\star}, \sum_{j=1}^{m} \pi_{j}^{\lambda_{n}}\right\rangle=0 .
$$

Finally, for any $y \in B(0,2 M) \cap \mathcal{K} \cap \operatorname{Dom}\left(f_{0}\right)$, from (35) we obtain

$$
\begin{aligned}
0 & \leq\left\langle y-x^{\lambda_{n}}, \sum_{j=1}^{m} \pi_{j}^{\lambda_{n}}+\tau^{\lambda_{n}}\right\rangle \\
& =\left\langle y-x^{\lambda_{n}}, \sum_{j=1}^{m} \pi_{j}^{\lambda_{n}}\right\rangle+\left\langle y-z^{\lambda_{n}}, \tau^{\lambda_{n}}\right\rangle+\left\langle z^{\lambda_{n}}-x^{\lambda_{n}}, \tau^{\lambda_{n}}\right\rangle \\
& \leq\left\langle y-x^{\lambda_{n}}, \sum_{j=1}^{m} \pi_{j}^{\lambda_{n}}\right\rangle+f_{0}(y)-f_{0}\left(z^{\lambda_{n}}\right)-\frac{\left\|x^{\lambda_{n}}-z^{\lambda_{n}}\right\|^{2}}{\lambda_{n}} \\
& \leq\left\langle y-x^{\lambda_{n}}, \sum_{j=1}^{m} \pi_{j}^{\lambda_{n}}\right\rangle+f_{0}(y)-f_{0}\left(z^{\lambda_{n}}\right) .
\end{aligned}
$$


Passing again to the limit with $n \rightarrow \infty$ yields

$$
\left\langle y-x^{\star}, \sum_{j=1}^{m} \pi_{j}^{\star}\right\rangle+f_{0}(y)-f_{0}\left(x^{\star}\right) \geq 0, \quad \forall y \in B(0,2 M) \cap \mathcal{K} .
$$

Substituting $y=y_{0}$ leads easily to the inequality

$$
f_{0}\left(x^{\star}\right) \leq \sum_{j=1}^{m} \phi_{j}\left(x^{\star}\right)+f_{0}\left(y_{0}\right)
$$

which thanks to $\left(H_{6}\right)$ implies $\left\|x^{\star}\right\| \leq M$. Having in mind the validity of (36) for any $y \in B(0,2 M) \cap \mathcal{K}$, we easily deduce (32). The proof is complete.

The problem (33) will be referred to as the economic equilibrium type problem. If additionally $\phi_{j}$ are positive homogeneous and $f_{0}=\sum_{j=1}^{m} \phi_{j}$ then (33) represents the variational formulation of the Arrow-Debreu model of economic equilibrium (cf. $[1,28]$ ). This variational inequality approach has been first introduced and studied in the setting of reflexive, separable Banach spaces in [34].

\section{Case $\phi_{j} \geq 0$}

In this section we improve the results of Proposition 4 by assuming that $\phi_{j} \geq 0$.

For any $\varepsilon>0$ introduce the data system on $(X \times \mathbb{R}) \times\left(X^{\star} \times \mathbb{R}\right)$ by

$$
\left.\begin{array}{l}
\tilde{f}_{j}^{\star}{ }^{\varepsilon}(\tau, q)=f_{j}^{\star}(\tau)+\varphi^{\varepsilon}(q), \tau \in \mathcal{K}^{\star}, q \in \mathbb{R}_{-} \\
\widetilde{f}_{0}{ }^{\varepsilon}(y, z)=f_{0}(y)+z+\operatorname{ind}_{[\varepsilon, \infty)}(z), y \in \mathcal{K}, z \in \mathbb{R}_{+} \\
\widetilde{\phi}_{j}^{\varepsilon}(y, z)=\phi_{j}(y)+s \max \left\{\left(z-\frac{1}{2} \varepsilon\right), \frac{\varepsilon}{2}\right\}, y \in \mathcal{K}, z \in \mathbb{R}_{+},
\end{array}\right\}
$$

where $\varphi^{\varepsilon}(q)=-\varepsilon(-q)^{1-\varepsilon}+\operatorname{ind}_{[-s, 0]}(q), q \in \mathbb{R}_{-}, s>0$ is a positive constant with $s \in\left(0, \frac{1}{m}\right)$.

Proposition 5 Assume that for each $j=1, \ldots, m$ the hypotheses below hold:

$\left(H_{1}\right) \quad 0 \in \operatorname{cl}\left(\operatorname{Dom}\left(f_{j}^{\star}\right)\right)$ and $\forall y \in \mathcal{K} \backslash\{0\} \exists \beta \geq 0: \beta y \in \operatorname{Int}\left(\operatorname{Dom}\left(f_{j}\right)\right)$

$\left(H_{2}^{\prime}\right) \quad \exists \rho_{j}>0, \exists M_{j}>0:\left\{\tau \in \mathcal{K}^{\star}:\left\langle\partial f_{j}^{\star}(\tau), \tau\right\rangle \cap\left(\mathbb{R}_{-}+\rho_{j}\right)=\emptyset\right\} \subset B\left(0, M_{j}\right)$

$\left(H_{3}^{\prime}\right) \quad \phi_{j}(x) \geq 0 \quad \forall x \in \mathcal{K}$

$\left(H_{4}\right) \quad \partial f_{j}(0)$ is locally compact

$\left(H_{5}\right) \quad \phi_{j}: \mathcal{K} \rightarrow \mathbb{R}_{+}$is weakly continuous on $\mathcal{K}$

$\left(H_{6}^{\prime}\right) \quad \exists \rho>0, \exists M>0:\left\{y \in \mathcal{K}: f_{0}(y) \leq \sum_{j=1}^{m} \phi_{j}(y)+f_{0}(0)+\rho\right\} \subset B(0, M)$

$\left(H_{7}\right) \quad\left(-\sum_{j=1}^{m} \partial f_{j}(0)\right) \cap \partial f_{0}(0)=\emptyset$. 
Then for each $\varepsilon>0$ there exists $\left(x^{\varepsilon},\left(\pi_{j}^{\varepsilon}\right),\left(r_{j}^{\varepsilon}\right),\left(\alpha_{j}^{\varepsilon}\right)\right) \in\left(\mathcal{K} \backslash\{0\} \times\left(\mathcal{K}^{\star}\right)^{m} \times\left(\mathbb{R}_{+}\right)^{m} \times\right.$ $\left(\mathbb{R}_{+}\right)^{m}$ ) such that

$$
\left.\begin{array}{c}
\pi_{j}^{\varepsilon} \in \partial f_{j}\left(\alpha_{j}^{\varepsilon} x^{\varepsilon}\right) \\
-\sum_{j=1}^{m} \pi_{j}^{\varepsilon} \in \partial f_{0}\left(x^{\varepsilon}\right) \\
-\left\langle x^{\varepsilon}, \pi_{j}^{\varepsilon}\right\rangle-\phi_{j}\left(x^{\varepsilon}\right)-\varepsilon\left(\frac{s}{2}+q_{j}^{\varepsilon}\right) \in \partial \operatorname{ind}_{\geq 0}\left(\alpha_{j}^{\varepsilon}\right) \\
-q_{j}^{\varepsilon}=(1-\varepsilon)^{1 / \varepsilon}\left(\frac{1}{\alpha_{j}^{\varepsilon}+r_{j}^{\varepsilon}}\right)^{1 / \varepsilon}, q_{j}^{\varepsilon} \in[-s, 0), \quad-r_{j}^{\varepsilon} \in \partial \operatorname{ind}_{[-s, 0]}\left(q_{j}^{\varepsilon}\right), r_{j}^{\varepsilon} \geq 0 .
\end{array}\right\}
$$

Proof Now we show that all the requirements of Proposition 4 are fulfilled when replacing $\left(f_{j}^{\star}, f_{0}, \phi_{j}\right)$ by $\left(\widetilde{f}_{j}^{\star \varepsilon}, \widetilde{f}_{0}^{\varepsilon}, \widetilde{\phi}_{j}^{\varepsilon}\right)$.

Let us begin with $\left(\mathrm{H}_{3}\right)$. It is easy to check that

$$
\tilde{\phi}_{j}^{\varepsilon}(y, z)=\phi_{j}(y)+s \max \left\{\left(z-\frac{1}{2} \varepsilon\right), \frac{\varepsilon}{2}\right\} \geq \frac{s}{2} \varepsilon>0, \forall(y, z) \in \mathcal{K} \times \mathbb{R}_{+},
$$

what desired.

To check that $\left(H_{2}\right)$ holds, let $y \in \partial f_{j}^{\star}(\tau)$ and $z \in \partial \varphi^{\varepsilon}(q)$. Hence $z=\varepsilon(1-$ $\left.\varepsilon)(-q)^{-\varepsilon}-r,-r \in \partial \operatorname{ind}_{[-s, 0)}(q)\right], r \geq 0, q \in[-s, 0)$. From the condition

$$
\begin{aligned}
0 & \geq\left\langle\partial \tilde{f}_{j}^{\star^{\varepsilon}}(\tau, q),(\tau, q)\right\rangle=\left\langle\partial f_{j}^{\star}(\tau), \tau\right\rangle+\partial \varphi^{\varepsilon}(q) q \\
& \geq\left\langle\partial f_{j}^{\star}(\tau), \tau\right\rangle+q\left[\varepsilon(1-\varepsilon)\left(-q^{\varepsilon}\right)^{-\varepsilon}-r\right] \geq\left\langle\partial f_{j}^{\star}(\tau), \tau\right\rangle-\varepsilon s\left(\frac{1}{s}\right)^{\varepsilon}
\end{aligned}
$$

we are led to

$$
\varepsilon s\left(\frac{1}{s}\right)^{\varepsilon} \geq\left\langle\partial f_{j}^{\star}(\tau), \tau\right\rangle
$$

Hence, by $\left(H_{2}^{\prime}\right)$ it follows that $\|\tau\| \leq M_{j}$ provided $\varepsilon>0$ is small enough. The boundedness of $q \in[-s, 0]$ is obvious.

Concerning $\left(\mathrm{H}_{6}\right)$, we wish to deduce that

$$
\left\{(y, z) \in \mathcal{K} \times\left(\mathbb{R}_{+}+\varepsilon\right): \widetilde{f}_{0}^{\varepsilon}(y, z) \leq \sum_{j=1}^{m} \widetilde{\phi}_{j}^{\varepsilon}(y, z)+\widetilde{f}_{0}^{\varepsilon}(0, \varepsilon)\right\}
$$

is bounded. Notice that this set can be rewritten equivalently as

$$
\left\{(y, z) \in \mathcal{K} \times\left(\mathbb{R}_{+}+\varepsilon\right): f_{0}(y)+z(1-s m) \leq \sum_{j=1}^{m} \phi_{j}(y)+f_{0}(0)+\varepsilon\left(1-\frac{s m}{2}\right)\right\} .
$$


So, by $\left(H_{6}^{\prime}\right)$ we get $\|y\| \leq M$. This in turn leads to

$$
z \leq \frac{1}{1-s m} \sup _{\substack{\|y\| \leq M \\ y \in \mathcal{K}}}\left\{\sum_{j=1}^{m} \phi_{j}(y)-f_{0}(y)+f_{0}(0)+\varepsilon\right\}
$$

as desired. The remaining assumptions of Proposition 4 are obviously satisfied. Therefore, we conclude the existence of

$$
\left(\left(x^{\varepsilon}, z^{\varepsilon}\right),\left(\pi_{j}^{\varepsilon}, q_{j}^{\varepsilon}\right),\left(\alpha_{j}^{\varepsilon}\right)\right) \in\left(\left(\mathcal{K} \times \mathbb{R}_{+} \times\left(\left(\mathcal{K}^{\star}\right)^{m} \times\left(\mathbb{R}_{-}\right)^{m}\right) \times\left(\mathbb{R}_{+}\right)^{m}\right)\right.
$$

such that

$$
\left.\begin{array}{c}
\alpha_{j}^{\varepsilon}\left(x^{\varepsilon}, z^{\varepsilon}\right) \in \partial{\widetilde{f_{j}^{*}}}^{\varepsilon}\left(\pi_{j}^{\varepsilon}, q_{j}^{\varepsilon}\right) \\
-\left\langle\left(x^{\varepsilon}, z^{\varepsilon}\right),\left(\pi_{j}^{\varepsilon}, q_{j}^{\varepsilon}\right)\right\rangle-\widetilde{\phi}_{j}^{\varepsilon}\left(x^{\varepsilon}, z^{\varepsilon}\right) \in \partial \operatorname{ind}_{\geq 0}\left(\alpha_{j}^{\varepsilon}\right) \\
-\sum_{j=1}^{m}\left(\pi_{j}^{\varepsilon}, q_{j}^{\varepsilon}\right) \in \partial \widetilde{f}_{0}^{\varepsilon}\left(x^{\varepsilon}, z^{\varepsilon}\right),
\end{array}\right\}
$$

or equivalently,

$$
\begin{gathered}
\alpha_{j}^{\varepsilon} x^{\varepsilon} \in \partial f_{j}^{\star}\left(\pi_{j}^{\varepsilon}\right), \quad \alpha_{j}^{\varepsilon} z^{\varepsilon} \in \partial \varphi^{\varepsilon}\left(q_{j}^{\varepsilon}\right)=\varepsilon(1-\varepsilon)\left(-q_{j}^{\varepsilon}\right)^{-\varepsilon}+\partial \operatorname{ind}_{[-s, 0]}\left(q_{j}^{\varepsilon}\right) \\
-\left\langle x^{\varepsilon}, \pi_{j}^{\varepsilon}\right\rangle-\phi_{j}\left(x^{\varepsilon}\right)-z^{\varepsilon}\left(s+q_{j}^{\varepsilon}\right)+\frac{s}{2} \varepsilon \in \partial \operatorname{ind}_{\geq 0}\left(\alpha_{j}^{\varepsilon}\right) \\
-\sum_{j=1}^{m} \pi_{j}^{\varepsilon} \in \partial f_{0}\left(x^{\varepsilon}\right), \quad-\sum_{j=1}^{m} q_{j}^{\varepsilon} \in 1+\partial \operatorname{ind}_{[\varepsilon,+\infty)}\left(z^{\varepsilon}\right) .
\end{gathered}
$$

From $(40)_{1}$ it follows that $-\sum_{j=1}^{m} q_{j}^{\varepsilon} \leq s m<1$. In view of $(40)_{3}$ it is only possible when $z^{\varepsilon}=\varepsilon$. Hence we obtain

$$
-q_{j}^{\varepsilon}=(1-\varepsilon)^{1 / \varepsilon}\left(\frac{1}{\alpha_{j}^{\varepsilon}+r_{j}^{\varepsilon}}\right)^{1 / \varepsilon}, \quad q_{j}^{\varepsilon} \in[-s, 0), \quad-r_{j}^{\varepsilon} \in \partial \operatorname{ind}_{[-s, 0]}\left(q_{j}^{\varepsilon}\right), \quad r_{j}^{\varepsilon} \geq 0,
$$

which completes the proof.

Another version of this result is useful when studying economic equilibrium problems. Namely, if we assume that $\operatorname{Dom}\left(f_{0}^{\star}\right) \cap\left(-\mathcal{K}^{\star}\right)$ is bounded and the cone $\mathcal{K}^{\star}$ is normal (for the characterization of normal cones we refer to [41]), i.e. there exists $\rho>0$ such that

$$
\|\tau+\pi\| \geq \rho(\|\tau\|+\|\pi\|), \quad \forall \tau, \pi \in \mathcal{K}^{\star},\left({ }^{1}\right)
$$

then in Proposition 5 the assumption $\left(H_{1}\right)$ can be considerably simplified and $\left(H_{2}\right)$ is redundant. Namely, the following result is available.

\footnotetext{
${ }^{1}$ This condition is equivalent to the well known normality property of $\mathcal{K}^{\star}$ defined as the boundedness of $\left(B_{X^{\star}}(0,1)+\mathcal{K}^{\star}\right) \cap\left(B_{X^{\star}}(0,1)-\mathcal{K}^{\star}\right)$ in $X^{\star}$.
} 
Proposition 6 Suppose that $\mathcal{K}^{\star}$ is normal and for any $j=1, \ldots, m$, the assumptions below are fulfilled:
$\left(H_{1}^{\prime}\right) \quad 0 \in \operatorname{cl}\left(\operatorname{Dom}\left(f_{j}^{\star}\right)\right)$
$\left(H_{3}^{\prime}\right) \quad \phi_{j}(x) \geq 0 \quad \forall x \in \mathcal{K}$
$\left(H_{4}\right) \quad \partial f_{j}(0)$ is locally compact
$\left(H_{5}\right) \quad \phi_{j}: \mathcal{K} \rightarrow \mathbb{R}_{+}$is weakly continuous on $\mathcal{K}$
$\left(H_{6}^{\prime}\right) \quad \exists \rho>0, \exists M>0:\left\{y \in \mathcal{K}: f_{0}(y) \leq \sum_{j=1}^{m} \phi_{j}(y)+f_{0}(0)+\rho\right\} \subset B(0, M)$
$\left(H_{7}\right) \quad\left(-\sum_{j=1}^{m} \partial f_{j}(0)\right) \cap \partial f_{0}(0)=\varnothing$
$\left(H_{8}\right) \quad \operatorname{Dom}\left(f_{0}^{\star}\right) \cap\left(-\mathcal{K}^{\star}\right) \subset B(0, C)$ for some $C>0$.

Then for each $\varepsilon>0$ there exists $\left(x^{\varepsilon},\left(\pi_{j}^{\varepsilon}\right),\left(\alpha_{j}^{\varepsilon}\right)\right) \in \mathcal{K} \backslash\{0\} \times\left(\mathcal{K}^{\star}\right)^{m} \times\left(\mathbb{R}_{+}\right)^{m}$ such that (38) holds.

Proof Let $R=\frac{C}{\rho^{m}}+1$ and $f_{j R}^{\star}(\cdot):=f_{j}^{\star}(\cdot)+\operatorname{ind}_{\overline{B(0, R)}}(\cdot), j=1, \ldots, m$. It is easy to show that $f_{0}, f_{j R}^{\star}$ and $\phi_{j}$ satisfy all the hypotheses of Proposition 5. Indeed, since the effective domain of $f_{j R}^{\star}$ is bounded, the effective domain of its conjugate $\left(f_{j R}^{\star}\right)^{\star}$ coincides with the whole $X$. Consequently, $\left(H_{1}\right)$ and $\left(H_{2}\right)$ hold immediately while $\left(H_{4}\right)$ is obviously fulfilled. Accordingly, by Proposition 5 there exists $\left(x^{R},\left(\pi_{j}^{R}\right),\left(\alpha_{j}^{R}\right),\right) \in$ $\mathcal{K} \times\left(\mathcal{K}^{\star}\right)^{m} \times\left(\mathbb{R}_{+}\right)^{m}$, such that (38) holds with $\partial f_{j}^{\star}$ replaced by $\partial f_{j R}^{\star}$.

Now, from $\left(H_{8}\right)$ it follows that $\left\|\sum_{j=1}^{m} \pi_{j}^{R}\right\| \leq C$ which by (42) implies that $\left\|\pi_{j}^{R}\right\| \leq$ $\frac{C}{\rho^{m}}$. Hence $\left\|\pi_{j}^{R}\right\|<R$ for each $j=1, \ldots, m$. Therefore the inclusion $(38)_{2}$ takes the form $\alpha_{j}^{R} x^{R} \in \partial f_{j R}^{\star}\left(\pi_{j}^{R}\right)=\partial f_{j}^{\star}\left(\pi_{j}^{R}\right), j=1, \ldots, m$. But this means that $\left(x^{R},\left(\pi_{j}^{R}\right),\left(\alpha_{j}^{R}\right)\right)$ is a solution of (38) in which only the basic data $f_{0}, f_{j}, \phi_{j}$ are involved.

\section{Economic Equilibrium Type Problem}

Now we are going to study the behavior of $\left(x^{\varepsilon},\left(\pi_{j}^{\varepsilon}\right),\left(\alpha_{j}^{\varepsilon}\right)\right)$ when $\varepsilon \rightarrow 0$ in the circumstances of Propositions 5 and 6.

Theorem 4 Assume that for each $j=1, \ldots, m$ the hypotheses below hold:

$\left(H_{1}\right) \quad 0 \in \operatorname{cl}\left(\operatorname{Dom}\left(f_{j}^{\star}\right)\right)$ and $\forall y \in \mathcal{K} \backslash\{0\} \exists \beta \geq 0: \beta y \in \operatorname{Int}\left(\operatorname{Dom}\left(f_{j}\right)\right)$

$\left(H_{2}^{\prime}\right) \quad \exists \rho_{j}>0, \exists M_{j}>0:\left\{\tau \in \mathcal{K}^{\star}:\left\langle\partial f_{j}^{\star}(\tau), \tau\right\rangle \cap\left(\mathbb{R}_{-}+\rho_{j}\right)=\emptyset\right\} \subset B\left(0, M_{j}\right)$

$\left(H_{3}^{\prime}\right) \quad \phi_{j}(x) \geq 0 \quad \forall x \in \mathcal{K}$

$\left(H_{4}\right) \quad \partial f_{j}(0)$ is locally compact

$\left(H_{5}\right) \quad \phi_{j}: \mathcal{K} \rightarrow \mathbb{R}_{+}$is weakly continuous on $\mathcal{K}$

$\left(H_{6}^{\prime}\right) \quad \exists \rho>0, \exists M>0:\left\{y \in \mathcal{K}: f_{0}(y) \leq \sum_{j=1}^{m} \phi_{j}(y)+f_{0}(0)+\rho\right\} \subset B(0, M)$

$\left(H_{7}\right) \quad\left(-\sum_{j=1}^{m} \partial f_{j}(0)\right) \cap \partial f_{0}(0)=\varnothing$

$\left(H_{9}\right) \quad f_{j}^{\infty}(y)+\phi_{j}(y)>0, \forall y \in \mathcal{K}$.

Then there exist disjoint $J, J_{0} \subset\{1, \ldots, m\}$ with $J \cup J_{0}=\{1, \ldots, m\}$ and $x \in \mathcal{K} \backslash 0$, $\left(\pi_{j}\right) \in\left(\mathcal{K}^{\star}\right)^{m},\left(\alpha_{j}\right)_{j \in J} \in \operatorname{Int}\left(\mathbb{R}_{+}\right)^{|J|},\left(\alpha_{j}\right)_{j \in J_{0}}=0 \in\left(\mathbb{R}_{+}\right)^{\left|J_{0}\right|}$, such that

$$
\left.\begin{array}{c}
\pi_{j} \in \partial f_{j}\left(\alpha_{j} x\right), \quad\left\langle x, \pi_{j}\right\rangle+\phi_{j}(x)=0, \quad j \in J \\
\pi_{j} \in \partial f_{j}(0), \quad\left\langle x, \pi_{j}\right\rangle+\phi_{j}(x) \geq 0, \quad j \in J_{0} \\
-\sum_{j=1}^{m} \pi_{j} \in \partial f_{0}(x) .
\end{array}\right\}
$$


Proof To establish the result, Proposition 5 will be used. By the boundedness of $\left\{x^{\varepsilon}\right\}$ and $\left\{\pi_{j}^{\varepsilon}\right\}$ one can extract sequences $\left\{x^{\varepsilon_{n}}\right\}$ and $\left\{\pi_{j}^{\varepsilon_{n}}\right\}, \varepsilon_{n} \rightarrow 0$ as $n \rightarrow \infty$, such that

$$
\begin{gathered}
x^{\varepsilon_{n}} \rightarrow x \quad \text { weakly in } X \\
\pi_{j}^{\varepsilon_{n}} \rightarrow \pi_{j} \quad \text { weakly in } X^{\star}, \quad j=1, \ldots, m .
\end{gathered}
$$

Set $J, J_{0}, J_{\infty} \subset\{1, \ldots, m\}$ as follows

$$
\begin{aligned}
j \in J & \Leftrightarrow \lim _{n \rightarrow \infty} \alpha_{j}^{\varepsilon_{n}}=\alpha_{j} \in(0, \infty) \\
j \in J_{0} & \Leftrightarrow \lim _{n \rightarrow \infty} \alpha_{j}^{\varepsilon_{n}}=0 \\
j \in J_{\infty} & \Leftrightarrow \lim _{n \rightarrow \infty} \alpha_{j}^{\varepsilon_{n}}=+\infty .
\end{aligned}
$$

Proceeding like in the proof of Proposition 3 leads to

$$
\lim _{n \rightarrow \infty}\left\langle x^{\varepsilon_{n}}, \pi_{j}^{\varepsilon_{n}}\right\rangle=\left\langle x, \pi_{j}\right\rangle, \quad j=1, \ldots, m .
$$

Taking into account $(38)_{3}$ we get

$$
\begin{aligned}
& \left\langle x, \pi_{j}\right\rangle+\phi_{j}(x)=0, \quad j \in J \cup J_{\infty} \\
& \left\langle x, \pi_{j}\right\rangle+\phi_{j}(x) \geq 0, \quad j \in J_{0} .
\end{aligned}
$$

The maximal monotonicity of $\partial f_{j}$ easily ensures (43) for $j \in J \cup J_{0}$.

It remains to show that $J_{\infty}=\emptyset$. Recall that the recession functional $f_{j}^{\infty}$ of $f_{j}$ is defined by $[9,43]$

$$
f_{j}^{\infty}(y)=\lim _{\lambda \rightarrow+\infty} \frac{1}{\lambda} f_{j}\left(\lambda y+y_{0}\right), \quad y \in X
$$

where $y_{0}$ is an arbitrarily chosen element of $\operatorname{Dom}\left(f_{j}\right)$. Consider the case $\alpha_{j}^{\varepsilon_{n}} \rightarrow \infty$, i.e. $j \in J_{\infty}$. According to $(38)_{2}$ we have

$$
f_{j}(y)-f_{j}\left(\alpha_{j}^{\varepsilon_{n}} x^{\varepsilon_{n}}\right) \geq\left\langle y-\alpha_{j}^{\varepsilon_{n}} x^{\varepsilon_{n}}, \pi_{j}^{\varepsilon_{n}}\right\rangle \quad \forall y \in X .
$$

Substituting $y \mapsto \alpha_{j}^{\varepsilon_{n}} y+y_{0}$ with $y_{0} \in \operatorname{Dom}\left(f_{j}\right)$ leads to

$$
\frac{1}{\alpha_{j}^{\varepsilon_{n}}} f_{j}\left(\alpha_{j}^{\varepsilon_{n}} y+y_{0}\right)-\frac{1}{\alpha_{j}^{\varepsilon_{n}}} f_{j}\left(\alpha_{j}^{\varepsilon_{n}} x^{\varepsilon_{n}}\right) \geq\left\langle y-x^{\varepsilon_{n}}, \pi_{j}^{\varepsilon_{n}}\right\rangle+\frac{1}{\alpha_{j}^{\varepsilon_{n}}}\left\langle y_{0}, \pi_{j}^{\varepsilon_{n}}\right\rangle \quad \forall y \in X .
$$

In view of

$$
\liminf _{n \rightarrow \infty} \frac{1}{\alpha_{j}^{\varepsilon_{n}}} f_{j}\left(\alpha_{j}^{\varepsilon_{n}} x^{\varepsilon_{n}}\right) \geq f_{j}^{\infty}(x)
$$

[9], passing to the limit with $n \rightarrow \infty$ allows the conclusion

$$
f_{j}^{\infty}(y)-f_{j}^{\infty}(x) \geq\left\langle y-x, \pi_{j}\right\rangle \quad \forall y \in X,
$$


or equivalently,

$$
\pi_{j} \in \partial f_{j}^{\infty}(x)
$$

Since $f_{j}^{\infty}$ is positive homogeneous, convex, lower semicontinuous, we have $f_{j}^{\infty}(x)=$ $\left\langle x, \pi_{j}\right\rangle$ which implies $f_{j}^{\infty}(x)+\phi_{j}(x)=0$ in the contrary to $\left(H_{9}\right)$. So, $J_{\infty}=\emptyset$, as desired. Observe that $\left(H_{7}\right)$ ensures that $x \neq 0$. This completes the proof of Theorem 4.

Proceeding like in the proof of Proposition 6 one can formulate another version of Theorem 4 in which $\operatorname{Dom}\left(f_{0}^{\star}\right) \cap\left(-\mathcal{K}^{\star}\right)$ is bounded and the cone $\mathcal{K}^{\star}$ is normal. Then $\left(H_{1}\right)$ can be weakened and replaced by $\left(H_{1}^{\prime}\right)$. Moreover, $\left(H_{2}^{\prime}\right)$ is redundant.

Theorem 5 Assume that $\mathcal{K}^{\star}$ is normal, i.e. there exists $\rho>0$ such that

$$
\|\tau+\pi\| \geq \rho(\|\tau\|+\|\pi\|), \quad \forall \tau, \pi \in \mathcal{K}^{\star}
$$

Moreover, assume that for each $j=1, \ldots, m$ the hypotheses below hold:

$\left(H_{1}^{\prime}\right) \quad 0 \in \operatorname{cl}\left(\operatorname{Dom}\left(f_{j}^{\star}\right)\right)$

$\left(H_{3}^{\prime}\right) \quad \phi_{j}(x) \geq 0 \quad \forall x \in \mathcal{K}$

$\left(H_{4}\right) \quad \partial f_{j}(0)$ is locally compact

$\left(H_{5}\right) \quad \phi_{j}: \mathcal{K} \rightarrow \mathbb{R}_{+}$is weakly continuous on $\mathcal{K}$

$\left(H_{6}^{\prime}\right) \quad \exists \rho>0, \exists M>0:\left\{y \in \mathcal{K}: f_{0}(y) \leq \sum_{j=1}^{m} \phi_{j}(y)+f_{0}(0)+\rho\right\} \subset B(0, M)$

$\left(H_{7}\right) \quad\left(-\sum_{j=1}^{m} \partial f_{j}(0)\right) \cap \partial f_{0}(0)=\varnothing$

$\left(H_{8}\right) \quad \operatorname{Dom}\left(f_{0}^{\star}\right) \cap\left(-\mathcal{K}^{\star}\right) \subset B(0, C)$ for some $C>0$

$\left(H_{9}\right) \quad f_{j}^{\infty}(y)+\phi_{j}(y)>0, \forall y \in \mathcal{K}$.

Then there exist disjoint $J, J_{0} \subset\{1, \ldots, m\}$ with $J \cup J_{0}=\{1, \ldots, m\}$ and $x \in \mathcal{K} \backslash 0$, $\left(\pi_{j}\right) \in\left(\mathcal{K}^{\star}\right)^{m},\left(\alpha_{j}\right)_{j \in J} \in \operatorname{Int}\left(\mathbb{R}_{+}\right)^{|J|},\left(\alpha_{j}\right)_{j \in J_{0}}=0 \in\left(\mathbb{R}_{+}\right)^{\left|J_{0}\right|}$, such that (43) holds.

\section{Economic Equilibrium Problem}

Throughout this chapter it will be assumed that $\mathcal{K}^{\star}$ is normal, i.e., (42) holds, $\phi_{j} \geq 0$ $j=1, \ldots, m$, are convex, positive homogeneous functions and

$$
f_{0}(y)=\sum_{j=1}^{m} \phi_{j}(y)+\operatorname{ind}_{\mathcal{K}}(y), \quad y \in \mathcal{K} .
$$

Since $f_{0}$ is positive homogeneous, lower semicontinuous and convex, there exists a convex, closed, nonempty set $\Delta^{\star} \subset X^{\star}, 0 \in \Delta^{\star}, \Delta^{\star} \supset \Delta^{\star}+\mathcal{K}^{\star}$, such that $f_{0}(y)=$ $\sup _{\tau \in \Delta^{\star}}\langle y, \tau\rangle, y \in X$.

Under the foregoing assumptions (43) can be seen as the variational formulation of the economic equilibrium problem as introduced in [34]. $f_{0}$ characterizes the total budget function while $f_{j}^{\star}$ and $\phi_{j}$ are dis-utility and budget functions corresponding to $j$ 's consumer, respectively. In (43), $-\pi_{j} \in-\mathcal{K}^{\star}, j=1, \ldots, m$, and $x \in \mathcal{K}$ are the commodity bundles and the price vector in the generalized Walrasian equilibrium, 
respectively. Equation (43) $)_{3}$ can be referred to as the market clearing condition because for strictly positive price vector $x$ the classical formulation of this condition can be easily deduced (cf. [34]). Obviously, we shall be looking for nontrivial price vector $x$, i.e., $x \neq 0$.

Unfortunately, Theorem 5 does not cover the case of economic equilibrium problem as introduced above. Indeed, (48) does not imply $\left(H_{6}^{\prime}\right)$. Moreover, due to $\phi_{j}(0)=0$ and $f_{j}^{\infty}(0)=0$, condition $\left(H_{9}\right)$ cannot be satisfied. It must be also emphasized that $\left(H_{4}\right)$ and $\left(H_{5}\right)$ are too strong in many important infinite dimensional applications.

To overcome the aforementioned inconveniences we start our investigations with the approximate model in which $f_{0}$ is replaced by $f_{0}^{\mu}$ where

$$
f_{0}^{\mu}(y)=\left(\sum_{j=1}^{m} \phi_{j}(y)\right)^{1+\mu}+\operatorname{ind}_{\mathcal{K}}(y), \quad y \in X, \mu>0 .
$$

Our main result of this section proceeds in several steps formulated in the form of propositions.

First result in this direction is based on Proposition 5.

Proposition 7 Let $\mathcal{K}^{\star}$ be normal. Moreover, assume that for each $j=1, \ldots, m$ the hypotheses below hold:

$$
\begin{aligned}
& \left(H_{1}\right) \quad 0 \in \operatorname{cl}\left(\operatorname{Dom}\left(f_{j}^{\star}\right)\right) \text { and } \forall y \in \mathcal{K} \backslash\{0\} \exists \beta \geq 0: \beta y \in \operatorname{Int}\left(\operatorname{Dom}\left(f_{j}\right)\right) \\
& \left(H_{2}^{\prime}\right) \quad \exists \rho_{j}>0, \exists M_{j}>0:\left\{\tau \in \mathcal{K}^{\star}:\left\langle\partial f_{j}^{\star}(\tau), \tau\right\rangle \cap\left(\mathbb{R}_{-}+\rho_{j}\right)=\emptyset\right\} \subset B\left(0, M_{j}\right) \\
& \left(H_{3}^{\prime \prime}\right) \quad \exists \gamma_{j}>0: \phi_{j}(y) \geq \gamma_{j}\|y\| \quad \forall y \in \mathcal{K} \\
& \left(H_{4}\right) \quad \partial f_{j}(0) \text { is locally compact } \\
& \left(H_{5}\right) \quad \phi_{j}: \mathcal{K} \rightarrow \mathbb{R}_{+} \text {is weakly continuous on } \mathcal{K} \\
& \left(H_{10}^{\prime}\right) \quad 0 \notin \partial f_{j}(0) .
\end{aligned}
$$

Then for each $\mu>0$ there exist $\left.\left(x,\left(\pi_{j}\right),\left(\alpha_{j}\right)\right) \in \mathcal{K} \backslash\{0\} \times\left(\mathcal{K}^{\star}\right)^{m} \times\left(\mathbb{R}_{+}\right)^{m}\right)$ such that

$$
\left.\begin{array}{c}
\pi_{j} \in \partial f_{j}\left(\alpha_{j} x\right) \\
-\sum_{j=1}^{m} \pi_{j} \in \partial f_{0}^{\mu}(x) \\
-\left\langle x, \pi_{j}\right\rangle-\phi_{j}(x) \in \partial \operatorname{ind}_{\geq 0}\left(\alpha_{j}\right)
\end{array}\right\}
$$

Proof First we check that under the foregoing assumptions, $\left(H_{6}^{\prime}\right)$ of Proposition 5 is satisfied. By $\left(H_{3}^{\prime \prime}\right)$ we get $\sum_{j=1}^{m} \phi_{j}(y) \geq \gamma\|y\|, \quad \forall y \in K, \quad \gamma=\sum_{j=1}^{m} \gamma_{j}>0$. Hence from $\left(\sum_{j=1}^{m} \phi_{j}(y)\right)^{1+\mu} \leq \sum_{j=1}^{m} \phi_{j}(y)+\varepsilon$ we obtain the estimate $\|y\| \leq \frac{1}{\gamma}$ provided that $\sum_{j=1}^{m} \phi_{j}(y) \leq 1$. For $\sum_{j=1}^{m} \phi_{j}(y)>1$ it follows

$$
\sum_{j=1}^{m} \phi_{j}(y) \leq\left[(1+\varepsilon)^{\frac{1}{\varepsilon}}\right]^{\frac{\varepsilon}{\mu}} \leq e
$$

giving rise to $\|y\| \leq \frac{e}{\gamma}, \varepsilon \in(0, \mu]$, as required. 
To check that $\left(H_{7}\right)$ of Proposition 5 holds let us notice that $\partial f_{0}^{\mu}(0) \subset \mathcal{K}^{\star}$. Assuming that $\tau \in\left(-\sum_{j=1}^{m} \partial f_{j}(0)\right) \cap \partial f_{0}^{\mu}(0) \subset\left(-\mathcal{K}^{\star}\right) \cap \mathcal{K}^{\star}=\{0\}$ we have $\tau=\sum_{j=1}^{m} \tau_{j}$ where $\tau_{j} \in \partial f_{j}(0) \subset \mathcal{K}^{\star}$ and $\sum_{j=1}^{m} \tau_{j}=0$. In view of (42) it follows that $\tau_{j}=0$. Consequently, $0 \in \partial f_{j}(0)$ for each $j=1, \ldots, m$, in contrary to $\left(H_{10}^{\prime}\right)$. Hence $\left(H_{7}\right)$ holds.

Since the remaining assumptions of Proposition 5 are satisfied immediately, there exists $\left(x^{\varepsilon},\left(\pi_{j}^{\varepsilon}\right),\left(\alpha_{j}^{\varepsilon}\right)\right)$ with $x^{\varepsilon} \neq 0$, such that

$$
\left.\begin{array}{c}
\pi_{j}^{\varepsilon} \in \partial f_{j}\left(\alpha_{j}^{\varepsilon} x^{\varepsilon}\right) \\
-\sum_{j=1}^{m} \pi_{j}^{\varepsilon} \in \partial f_{0}^{\mu}\left(x^{\varepsilon}\right) \\
-\left\langle x^{\varepsilon}, \pi_{j}^{\varepsilon}\right\rangle-\phi_{j}\left(x^{\varepsilon}\right)-\varepsilon\left(\frac{s}{2}+q_{j}^{\varepsilon}\right) \in \partial \operatorname{ind}_{\geq 0}\left(\alpha_{j}^{\varepsilon}\right) \\
-q_{j}^{\varepsilon}=(1-\varepsilon)^{1 / \varepsilon}\left(\frac{1}{\alpha_{j}^{\varepsilon}+r_{j}^{\varepsilon}}\right)^{1 / \varepsilon}, q_{j}^{\varepsilon} \in[-s, 0), \quad-r_{j}^{\varepsilon} \in \partial \operatorname{ind}_{[-s, 0]}\left(q_{j}^{\varepsilon}\right), \quad r_{j}^{\varepsilon} \geq 0 .
\end{array}\right\}
$$

Now we proceed to show the existence of a nontrivial solution $x \neq 0$ of (50). By the boundedness argument we can suppose that for a subsequence $\varepsilon_{n} \rightarrow 0$, there exist $x \in \mathcal{K}$ and $\pi_{j} \in \mathcal{K}^{\star}$ with $x^{\varepsilon_{n}} \rightarrow x$ weakly in $X$ and $\pi_{j}^{\varepsilon_{n}} \rightarrow \pi_{j}$ weakly in $X^{\star}$, $j=1, \ldots, m$.

First we claim that $\left\|x^{\varepsilon_{n}}\right\| \geq \delta$ for some positive $\delta>0$. Indeed, assuming on the contrary that $x^{\varepsilon_{n}} \rightarrow 0$ strongly in $X$, by the maximal monotonicity of $\partial f_{0}^{\mu}$ we are led to $-\sum_{j=1}^{m} \pi_{j} \in \partial f_{0}^{\mu}(0) \subset \mathcal{K}^{\star}$ which implies $\pi_{j}=0, j=1, \ldots, m$.

Set $\tilde{x}^{\varepsilon_{n}}=\frac{x^{\varepsilon_{n}}}{\left\|x^{\varepsilon_{n}}\right\|}, \tilde{\alpha}_{j}^{\varepsilon_{n}}=\left\|x^{\varepsilon_{n}}\right\| \alpha_{j}^{\varepsilon_{n}}$.

Case $\tilde{\alpha}_{j}^{\varepsilon_{n}} \rightarrow \infty$. This implies $\alpha_{j}^{\varepsilon_{n}} \rightarrow \infty$ and by $(51)_{4}$ we are led to $q_{j}^{\varepsilon_{n}} \rightarrow 0$. By $\left(H_{3}^{\prime \prime}\right)$ we then obtain

$$
\begin{aligned}
0 & <\frac{1}{2} \gamma_{j} \leq \phi_{j}\left(\tilde{x}^{\varepsilon_{n}}\right)+\frac{\varepsilon_{n}}{\left\|x^{\varepsilon_{n}}\right\|}\left(\frac{s}{2}+q_{j}^{\varepsilon_{n}}\right)=-\left\langle\tilde{x}^{\varepsilon_{n}}, \pi_{j}^{\varepsilon_{n}}\right\rangle \\
& \leq \frac{m_{j}}{\tilde{\alpha}_{j}^{\varepsilon_{n}}}+\frac{1}{\tilde{\alpha}_{j}^{\varepsilon_{n}}} f_{j}^{\star}(\tau)+\|\tau\|\left\|\tilde{x}^{\varepsilon_{n}}\right\|, \quad \forall \tau \in \operatorname{Dom}\left(f_{j}^{\star}\right)
\end{aligned}
$$

for sufficiently large $n$. Letting $n \rightarrow \infty$ implies $\frac{1}{2} \gamma_{j} \leq 0$. The contradiction.

Case $\tilde{\alpha}_{j}^{\varepsilon_{n}} \rightarrow 0$. In such a case we are led to $0 \in \partial f_{j}(0)$ which contradicts $\left(H_{10}^{\prime}\right)$.

Case $\lim _{n \rightarrow \infty} \tilde{\alpha}_{j}^{\varepsilon_{n}}=\tilde{\alpha}_{j} \in(0,+\infty)$ for each $j=1, \ldots, m$. From $\pi_{j}^{\varepsilon_{n}} \in \partial f_{j}\left(\tilde{\alpha}_{j}^{\varepsilon_{n}} \tilde{x}^{\varepsilon_{n}}\right)$ we get easily $\lim \inf _{n \rightarrow \infty}\left\langle\tilde{x}^{\varepsilon_{n}}, \pi_{j}^{\varepsilon_{n}}\right\rangle \geq 0$ which leads to $\lim _{n \rightarrow \infty}\left\langle\tilde{x}^{\varepsilon_{n}}, \pi_{j}^{\varepsilon_{n}}\right\rangle=0, j=1, \ldots, m$. Further on, according to $(51)_{2}$ it follows

$$
\left.\left\|x^{\varepsilon_{n}}\right\|^{\mu} f_{0}^{\mu}\left(\tilde{x}^{\varepsilon}\right)\right)+\frac{1}{\left\|x^{\varepsilon_{n}}\right\|}\left(f_{0}^{\mu}\right)^{\star}\left(-\sum_{j=1}^{m} \pi_{j}^{\varepsilon_{n}}\right)=\left\langle\tilde{x}^{\varepsilon_{n}},-\sum_{j=1}^{m} \pi_{j}^{\varepsilon_{n}}\right\rangle
$$

giving rise to

$$
\lim _{n \rightarrow \infty} \frac{1}{\left\|x^{\varepsilon_{n}}\right\|}\left(f_{0}^{\mu}\right)^{\star}\left(-\sum_{j=1}^{m} \pi_{j}^{\varepsilon_{n}}\right)=0
$$


On the other hand, by $(51)_{3}$ and $(51)_{4}$,

$$
\begin{aligned}
& \left.\left\|x^{\varepsilon_{n}}\right\|^{\mu} f_{0}^{\mu}\left(\tilde{x}^{\varepsilon}\right)\right)+\frac{1}{\left\|x^{\varepsilon_{n}}\right\|}\left(f_{0}^{\mu}\right)^{\star}\left(-\sum_{j=1}^{m} \pi_{j}^{\varepsilon_{n}}\right) \\
& =\left\langle\tilde{x}^{\varepsilon_{n}},-\sum_{j=1}^{m} \pi_{j}^{\varepsilon_{n}}\right\rangle=\sum_{j=1}^{m} \phi_{j}\left(\tilde{x}^{\varepsilon_{n}}\right)+\frac{\varepsilon_{n}}{\left\|x^{\varepsilon_{n}}\right\|}\left(m \frac{s}{2}+\sum_{j=1}^{m} q_{j}^{\varepsilon_{n}}\right) .
\end{aligned}
$$

Since $\tilde{\alpha}_{j}>0$, so $\alpha_{j}^{\varepsilon_{n}} \rightarrow+\infty, j=1, \ldots, m$. In view of $(51)_{4}$ this implies $\sum_{j=1}^{m} q_{j}^{\varepsilon_{n}} \rightarrow 0$ which thanks to $\left(H_{3}^{\prime \prime}\right)$ leads to

$$
\begin{aligned}
\left.\left\|x^{\varepsilon_{n}}\right\|^{\mu} f_{0}^{\mu}\left(\tilde{x}^{\varepsilon}\right)\right)+\frac{1}{\left\|x^{\varepsilon_{n}}\right\|}\left(f_{0}^{\mu}\right)^{\star}\left(-\sum_{j=1}^{m} \pi_{j}^{\varepsilon_{n}}\right) & =\left\langle\tilde{x}^{\varepsilon_{n}},-\sum_{j=1}^{m} \pi_{j}^{\varepsilon_{n}}\right\rangle \\
& =\sum_{j=1}^{m} \phi_{j}\left(\tilde{x}^{\varepsilon_{n}}\right)+\frac{\varepsilon_{n}}{\left\|x^{\varepsilon_{n}}\right\|}\left(m \frac{s}{2}+\sum_{j=1}^{m} q_{j}^{\varepsilon_{n}}\right) \\
& \geq \sum_{j=1}^{m} \gamma_{j} .
\end{aligned}
$$

Therefore, letting $n \rightarrow \infty$ leads again to the contradiction $0 \geq \sum_{j=1}^{m} \gamma_{j}$. The proof of the claim that $\left\|x^{\varepsilon_{n}}\right\| \geq \delta$ is complete.

The condition $\left\|x^{\varepsilon_{n}}\right\| \geq \delta$ allows to establish (50) by repeating the reasoning already presented. Therefore the details will be omitted here. The proof of Proposition 7 is complete.

Now we are ready to examine the case $\mu \rightarrow 0$.

Proposition 8 Let $\mathcal{K}^{\star}$ be normal. Moreover, assume that for $j=1, \ldots, m$, the hypotheses below hold:

$$
\begin{array}{ll}
\left(H_{1}\right) & 0 \in \operatorname{cl}\left(\operatorname{Dom}\left(f_{j}^{\star}\right)\right) \text { and } \forall y \in \mathcal{K} \backslash\{0\} \exists \beta \geq 0: \beta y \in \operatorname{Int}\left(\operatorname{Dom}\left(f_{j}\right)\right) \\
\left(H_{2}^{\prime}\right) & \exists \rho_{j}>0, \exists M_{j}>0:\left\{\tau \in \mathcal{K}^{\star}:\left\langle\partial f_{j}^{\star}(\tau), \tau\right\rangle \cap\left(\mathbb{R}_{-}+\rho_{j}\right)=\emptyset\right\} \subset B\left(0, M_{j}\right) \\
\left(H_{3}^{\prime \prime}\right) & \exists \gamma_{j}>0: \phi_{j}(y) \geq \gamma_{j}\|y\| \quad \forall y \in \mathcal{K} \\
\left(H_{4}\right) & \partial f_{j}(0) \text { is locally compact } \\
\left(H_{5}\right) & \phi_{j}: \mathcal{K} \rightarrow \mathbb{R}_{+} \text {is weakly continuous on } \mathcal{K} \\
\left(H_{7}^{\prime}\right) & \left(-\sum_{j=1}^{m} \partial f_{j}(0)\right) \cap \Delta^{\star}=\emptyset \\
\left(H_{10}^{\prime}\right) & 0 \notin \partial f_{j}(0) .
\end{array}
$$

Then there exist $\left(x,\left(\pi_{j}\right),\left(\alpha_{j}\right)\right) \in \mathcal{K} \backslash\{0\} \times\left(\mathcal{K}^{\star}\right)^{m} \times\left(\mathbb{R}_{+}\right)^{m}$ and $r \in(0,1]$ such that

$$
\left.\begin{array}{c}
\pi_{j} \in \partial f_{j}\left(\alpha_{j} x\right), \quad j=1, \ldots, m \\
-\sum_{j=1}^{m} \pi_{j} \in r \partial f_{0}(x) \\
-\phi_{j}(x) \in \partial \operatorname{ind}_{\geq 0}\left(\alpha_{j}\right), \quad j=1, \ldots, m .
\end{array}\right\}
$$


Proof By Proposition 7 we are allowed to suppose that for a sequence $\mu_{n} \rightarrow 0$ there exists $\left.\left(x^{\mu_{n}},\left(\pi_{j}^{\mu_{n}}\right),\left(\alpha_{j}^{\mu_{n}}\right)\right) \in \mathcal{K} \backslash\{0\} \times\left(\mathcal{K}^{\star}\right)^{m} \times\left(\mathbb{R}_{+}\right)^{m}\right)$ such that

$$
\left.\begin{array}{c}
0 \in \partial f_{0}^{\mu_{n}}\left(x^{\mu_{n}}\right)+\sum_{j=1}^{m} \partial f_{j}\left(\alpha_{j}^{\mu_{n}} x^{\mu_{n}}\right) \\
\pi_{j}^{\mu_{n}} \in \partial f_{j}\left(\alpha_{j}^{\mu_{n}} x^{\mu_{n}}\right) \\
-\sum_{j=1}^{m} \pi_{j}^{\mu_{n}} \in \partial f_{0}^{\mu_{n}}\left(x^{\mu_{n}}\right) \\
-\left\langle x^{\mu_{n}}, \pi_{j}^{\mu_{n}}\right\rangle-\phi_{j}\left(x^{\mu_{n}}\right) \in \partial \operatorname{ind}_{\geq 0}\left(\alpha_{j}^{\mu_{n}}\right)
\end{array}\right\}
$$

and $x^{\mu_{n}} \rightarrow x, \pi_{j}^{\mu_{n}} \rightarrow \pi_{j}$ weakly in $X$ and $X^{\star}$, respectively.

Consider two cases.

Case $1\left\|x^{\mu_{n}}\right\| \geq \delta>0$. By $\left(H_{3}^{\prime \prime}\right)$ we ensure the boundedness of $\left(\alpha_{j}^{\mu_{n}}\right)$ and therefore we are allowed to assume that $\alpha_{j}^{\mu_{n}} \rightarrow \alpha_{j} \in \mathbb{R}_{+}, j=1, \ldots, m$. The method that has been already explained allows the conclusion $\lim _{n \rightarrow \infty}\left\langle x^{\mu_{n}}, \pi_{j}^{\mu_{n}}\right\rangle=\left\langle x, \pi_{j}\right\rangle, j=1, \ldots, m$, which by the maximal monotonicity argument leads to $-\sum_{j=1}^{m} \pi_{j} \in \partial f_{0}(x) \subset \Delta^{\star}$ and $\pi_{j} \in \partial f_{j}\left(\alpha_{j} x\right)$ for each $j=1, \ldots, m$. In view of $\left(H_{7}^{\prime}\right)$ it follows that $x \neq 0$ and that for at least one $j \in\{1, \ldots, m\}, \alpha_{j}>0 .\left(H_{5}\right)$ ensures the validity of $(55)_{3}$. Finally we conclude that $\left.\left(x,\left(\pi_{j}\right),\left(\alpha_{j}\right)\right) \in \mathcal{K} \backslash\{0\} \times\left(\mathcal{K}^{\star}\right)^{m} \times\left(\mathbb{R}_{+}\right)^{m}\right)$ fulfills (55) with $r=1$.

Case $2\left\|x^{\mu_{n}}\right\| \rightarrow 0$ as $n \rightarrow \infty$. Introduce $\tilde{x}^{\mu_{n}}=\frac{1}{\left\|x^{\mu_{n}}\right\|} x^{\mu_{n}}$ and $\tilde{\alpha}_{j}^{\mu_{n}}=\alpha_{j}^{\mu_{n}}\left\|x^{\mu_{n}}\right\|, j=$ $1, \ldots, m$. By the boundedness argument one can suppose that $\tilde{x}^{\mu_{n}} \rightarrow \tilde{x}$ weakly in $X$ and $\left(\left\|x^{\mu_{n}}\right\|\right)^{\mu_{n}} \rightarrow r$ with $r \in[0,1]$.

The boundedness of $\left(\tilde{\alpha}_{j}^{\mu_{n}}\right)$ follows from the estimate

$$
0<\gamma_{j} \leq \phi_{j}\left(\tilde{x}^{\mu_{n}}\right)=-\left\langle\tilde{x}^{\mu_{n}}, \pi_{j}^{\mu_{n}}\right\rangle \leq \frac{m_{j}}{\tilde{\alpha}_{j}^{\mu_{n}}}+\frac{1}{\tilde{\alpha}_{j}^{\mu_{n}}} f_{j}^{\star}(\tau)+\|\tau\|\left\|\tilde{x}^{\mu_{n}}\right\|, \quad \forall \tau \in \operatorname{Dom}\left(f_{j}^{\star}\right)
$$

Indeed, assuming that $\tilde{\alpha}_{j}^{\mu_{n}} \rightarrow+\infty$ as $n \rightarrow \infty$ leads to

$$
0<\gamma_{j} \leq\|\tau\|, \quad \forall \tau \in \operatorname{Dom}\left(f_{j}^{\star}\right) .
$$

which thanks to $\left(H_{1}\right)$ implies the contradiction $\gamma_{j} \leq 0$. Therefore one can suppose that $\tilde{\alpha}_{j}^{\mu_{n}} \rightarrow \tilde{\alpha}_{j}$ for each $j=1, \ldots, m$.

According to $(56)_{3}$ it follows

$$
\left(\left\|x^{\mu_{n}}\right\|\right)^{\mu_{n}}\left[\left(f_{0}(y)\right)^{1+\mu_{n}}-\left(f_{0}\left(\tilde{x}^{\mu_{n}}\right)\right)^{1+\mu_{n}}\right] \geq\left\langle y-\tilde{x}^{\mu_{n}},-\sum_{j=1}^{m} \pi_{j}^{\mu_{n}}\right\rangle, \quad \forall y \in \mathcal{K},
$$

which by substituting $y \mapsto \tilde{x}$ and letting $n \rightarrow \infty$ yields

$$
\limsup _{n \rightarrow \infty}\left\langle\tilde{x}^{\mu_{n}}, \sum_{j=1}^{m} \pi_{j}^{\mu_{n}}\right\rangle \leq\left\langle\tilde{x}, \sum_{j=1}^{m} \pi_{j}\right\rangle
$$


As in the proof of Proposition 6 from $\pi_{j}^{\mu_{n}} \in \partial f_{j}\left(\tilde{\alpha}_{j} \tilde{x}^{\mu_{n}}\right)$ we get

$$
\liminf _{n \rightarrow \infty}\left\langle\tilde{x}^{\mu_{n}}, \pi_{j}^{\mu_{n}}\right\rangle \geq\left\langle\tilde{x}, \pi_{j}\right\rangle, \quad j=1, \ldots, m .
$$

Combining this with (59) yields

$$
\lim _{n \rightarrow \infty}\left\langle\tilde{x}^{\mu_{n}}, \pi_{j}^{\mu_{n}}\right\rangle=\left\langle\tilde{x}, \pi_{j}\right\rangle, \quad j=1, \ldots, m .
$$

Thus maximal monotonicity of $\partial f_{j}$ allows the conclusion that $\pi_{j} \in \partial f_{j}\left(\tilde{\alpha}_{j} \tilde{x}\right) j=$ $1, \ldots, m$.

Now passing to the limit in (58) leads to

$$
r\left(f_{0}(y)-f_{0}(\tilde{x}) \geq\left\langle y-\tilde{x},-\sum_{j=1}^{m} \pi_{j}\right\rangle, \quad \forall y \in \mathcal{K} .\right.
$$

Thus the existence of the desired $\tilde{x}$ and $r$ has been established. It remains to show that $\tilde{x} \neq 0$ and $r>0$.

Indeed, if $r=0$ then $-\sum_{j=1}^{m} \pi_{j} \in \mathcal{K}^{\star}$. On the other hand, $\sum_{j=1}^{m} \pi_{j} \in \mathcal{K}^{\star}$ which in view of $\mathcal{K}^{\star} \cap\left(-\mathcal{K}^{\star}\right)=\{0\}$ yields $\sum_{j=1}^{m} \pi_{j}=0$ and consequently, because $\mathcal{K}^{\star}$ is normal we have $\pi_{j}=0$ for each $j=1, \ldots, m$. Thus $0 \in \partial f_{j}\left(\tilde{\alpha}_{j} \tilde{x}\right), j=1, \ldots, m$, which by $\left(H_{10}^{\prime}\right)$ means that $\tilde{x} \neq 0$ and $\tilde{\alpha}_{j}>0$ for each $j=1, \ldots, m$. Further on, from $(56)_{4}$ we get

$$
0<\gamma_{j} \leq \phi_{j}\left(\tilde{x}^{\mu_{n}}\right)=-\left\langle\tilde{x}^{\mu_{n}}, \pi_{j}^{\mu_{n}}\right\rangle
$$

which by passing to the limit leads to the contradiction $\gamma_{j} \leq 0$. This contradiction ensures that $r>0$.

Let $\tilde{x}=0$. According to (61), $f_{0}(y) \geq\left\langle y,-\frac{1}{r} \sum_{j=1}^{m} \pi_{j}\right\rangle$ for all $y \in X$ which is equivalent to $-\frac{1}{r} \sum_{j=1}^{m} \pi_{j} \in \Delta^{\star}$. But $0 \in \Delta^{\star}$, so $-\sum_{j=1}^{m} \partial f_{j}(0) \ni-\sum_{j=1}^{m} \pi_{j} \in r \Delta^{\star} \subset \Delta^{\star}$ in the contrary to $\left(H_{7}^{\prime}\right)$. The claim follows.

Accordingly we arrive at $(55)_{2}$. The validity of $(55)_{3}$ is obvious in view of $\left(H_{5}\right)$ and (60). This completes the proof of Proposition 8.

Remark 1 Since $f_{0}^{\star}=\operatorname{ind}_{\Delta^{\star}}$ the assumption $\left(H_{8}\right)$ of Proposition 6 takes the form $\left(H_{8}^{\prime}\right) \quad \exists C>0: \Delta^{\star} \cap\left(-\mathcal{K}^{\star}\right) \subset B(0, C)$.

By proceeding like in the proof of Proposition 6 we arrive at the result.

Proposition 9 Let $\mathcal{K}^{\star}$ be normal. Moreover, let for each $j=1, \ldots, m$, the hypotheses below hold:

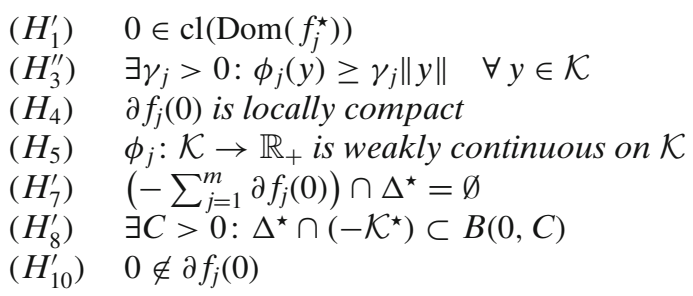

Then there exist $\left(x,\left(\pi_{j}\right),\left(\alpha_{j}\right)\right) \in \mathcal{K} \backslash\{0\} \times\left(\mathcal{K}^{\star}\right)^{m} \times\left(\mathbb{R}_{+}\right)^{m}$ and $r \in(0,1]$ such that (55) holds. 
Remark 2 Thanks to the positive homogeneity of $\phi_{j}(\cdot), j=1, \ldots, m$, and $f_{0}(\cdot)$ we are allowed to suppose that $x$ in Proposition 9 lies on a sphere with a radius $c>0$ which may be specified arbitrarily when necessary.

Remark 3 The assumption $\left(H_{3}^{\prime \prime}\right)$ is too restrictive in many applications. It can be replaced by a weaker one

$$
\left(H_{3}^{\prime \prime \prime}\right) \quad \exists \gamma>0: \sum_{j=1}^{m} \phi_{j}(y) \geq \gamma\|y\| \quad \forall y \in \mathcal{K} .
$$

To have the Lagrange multipliers $\left(\alpha_{j}\right)$ finite (i.e. $\left.\alpha_{j}<+\infty\right)$ we shall need a weaker version of $\left(H_{9}\right)$, namely

$$
\left(H_{9}^{\prime}\right) \quad \phi_{j}(y)+f_{j}^{\infty}(y)>0 \forall y \in \mathcal{K} \backslash\{0\} .
$$

We summarize foregoing remarks in the form of the following result.

Proposition 10 Let $\mathcal{K}^{\star}$ be normal. Moreover, assume that for each $j \in\{1, \ldots, m\}$ the hypotheses below hold:

$$
\begin{array}{ll}
\left(H_{1}^{\prime}\right) & 0 \in \operatorname{cl}\left(\operatorname{Dom}\left(f_{j}^{\star}\right)\right) \\
\left(H_{3}^{\prime \prime \prime}\right) & \exists \gamma>0: \sum_{j=1}^{m} \phi_{j}(y) \geq \gamma\|y\| \quad \forall y \in \mathcal{K} \\
\left(H_{4}\right) & \partial f_{j}(0) \text { is locally compact } \\
\left(H_{5}\right) & \phi_{j}: \mathcal{K} \rightarrow \mathbb{R}_{+} \text {is weakly continuous on } \mathcal{K} \\
\left(H_{7}^{\prime}\right) & \left(-\sum_{j=1}^{m} \partial f_{j}(0)\right) \cap \Delta^{\star}=\emptyset \\
\left(H_{8}^{\prime}\right) & \exists C>0: \Delta^{\star} \cap\left(-\mathcal{K}^{\star}\right) \subset B(0, C) \\
\left(H_{9}^{\prime}\right) & \phi_{j}(y)+f_{j}^{\infty}(y)>0 \forall y \in \mathcal{K} \backslash\{0\} \\
\left(H_{10}^{\prime}\right) & 0 \notin \partial f_{j}(0) .
\end{array}
$$

Then there exist $\left(x,\left(\pi_{j}\right),\left(\alpha_{j}\right)\right) \in \mathcal{K} \times\left(\mathcal{K}^{\star}\right)^{m} \times\left(\mathbb{R}_{+}\right)^{m},\|x\|=1$, and $r \in(0,1]$ such that

$$
\left.\begin{array}{c}
\pi_{j} \in \partial f_{j}\left(\alpha_{j} x\right), \quad j=1, \ldots, m \\
-\sum_{j=1}^{m} \pi_{j} \in r \partial f_{0}(x) \\
-\phi_{j}(x) \in \partial \operatorname{ind}_{\geq 0}\left(\alpha_{j}\right), \quad j=1, \ldots, m .
\end{array}\right\}
$$

Proof To apply Proposition 9 replace $\phi_{j}(\cdot)$ by $\phi_{j}^{\varepsilon}(y)=\phi_{j}(y)+\varepsilon\|y\|, y \in \mathcal{K}$. Then there exist $\left(x^{\varepsilon},\left(\pi_{j}^{\varepsilon}\right),\left(\alpha_{j}^{\varepsilon}\right)\right) \in \mathcal{K} \times\left(\mathcal{K}^{\star}\right)^{m} \times\left(\mathbb{R}_{+}\right)^{m},\left\|x^{\varepsilon}\right\|=1$ and $r^{\varepsilon} \in(0,1]$ such that (55) holds, i.e.

$$
\left.\begin{array}{c}
\pi_{j}^{\varepsilon} \in \partial f_{j}\left(\alpha_{j}^{\varepsilon} x\right), \quad j=1, \ldots, m \\
-\sum_{j=1}^{m} \pi_{j}^{\varepsilon} \in r^{\varepsilon} \partial f_{0}^{\varepsilon}\left(x^{\varepsilon}\right) \\
-\left\langle x^{\varepsilon}, \pi_{j}^{\varepsilon}\right\rangle-\phi_{j}\left(x^{\varepsilon}\right)-\varepsilon\left\|x^{\varepsilon}\right\| \in \partial \operatorname{ind}_{\geq 0}\left(\alpha_{j}^{\varepsilon}\right), \quad j=1, \ldots, m,
\end{array}\right\}
$$

where $f_{0}^{\varepsilon}(\cdot)=f_{0}(\cdot)+m \varepsilon\|\cdot\|$. By the boundedness we can assume that for a sequence $\varepsilon_{n} \rightarrow 0$ as $n \rightarrow \infty, x^{\varepsilon_{n}} \rightarrow x$ and $\pi_{j}^{\varepsilon_{n}} \rightarrow \pi_{j}$ weakly in $X$ and $X^{\star}$, respectively, 
and $r^{\varepsilon_{n}} \rightarrow r$. On the way already presented we arrive at $\lim _{n \rightarrow \infty}\left\langle x^{\varepsilon_{n}}, \pi_{j}^{\varepsilon_{n}}\right\rangle=\left\langle x, \pi_{j}\right\rangle$, $j=1, \ldots, m$. Thus $\pi_{j} \in \partial f_{j}\left(\alpha_{j} x\right)$ provided that $j \in J=\left\{i \in\{1, \ldots, m\}: \alpha_{i}^{\varepsilon_{n}} \rightarrow \alpha_{i} \in\right.$ $\left.\mathbb{R}_{+}\right\}$. If $j \in J_{\infty}=\left\{i \in\{1, \ldots, m\}: \alpha_{i}^{\varepsilon_{n}} \rightarrow+\infty\right\}$ then from

$$
0 \geq-\varepsilon_{n}\left\|x^{\varepsilon_{n}}\right\|=\phi_{j}\left(x^{\varepsilon_{n}}\right)+\frac{1}{\alpha_{j}^{\varepsilon_{n}}} f_{j}\left(\alpha_{j}^{\varepsilon} x^{\varepsilon_{n}}\right)+\frac{1}{\alpha_{j}^{\varepsilon_{n}}} f_{j}^{\star}\left(\pi_{j}^{\varepsilon_{n}}\right), \quad j=1, \ldots, m,
$$

it results

$$
0 \geq \phi_{j}(x)+f_{j}^{\infty}(x)
$$

Moreover, passing to the limit in $(63)_{2}$ yields

$$
r f_{0}(y)-r f_{0}(x) \geq\left\langle y-x,-\sum_{j=1}^{m} \pi_{j}\right\rangle \quad \forall y \in \mathcal{K} .
$$

Now consider two possible cases.

Case $1 J_{\infty} \neq \emptyset$. This condition by $\left(H_{9}^{\prime}\right)$ and (64) implies $x=0$, and consequently, $\pi_{j} \in \partial f_{j}(0)$ for each $j \in J$.

If $r=0$ then from (65) we get $-\sum_{j=1}^{m} \pi_{j} \in \mathcal{K}^{\star}$. Since $\sum_{j=1}^{m} \pi_{j} \in \mathcal{K}^{\star}$, in view of $\mathcal{K}^{\star} \cap$ $\left(-\mathcal{K}^{\star}\right)=\{0\}$ it follows $\sum_{j=1}^{m} \pi_{j}=0$ and consequently, $\left(\mathcal{K}^{\star}\right.$ is normal $), \pi_{j}=0$ for each $j=1, \ldots, m$. Hence, $0 \in \partial f_{j}(0)$ for each $j \in J$ which in view of $\left(H_{10}^{\prime}\right)$ is impossible if $J$ is not empty. Thus $J=\emptyset$. But this implies that $\alpha_{j}^{\varepsilon_{n}}>0$ for sufficiently large $n$ and each $j=1, \ldots, m$. However, this can happen when $r^{\varepsilon_{n}}=1$. Therefore, from $\left(H_{3}^{\prime \prime \prime}\right)$ it follows

$$
\gamma \leq \sum_{j=1}^{m} \phi_{j}\left(x^{\varepsilon_{n}}\right)+m \varepsilon_{n}=f_{0}\left(x^{\varepsilon_{n}}\right)+m \varepsilon_{n}=\left\langle x^{\varepsilon_{n}},-\sum_{j=1}^{m} \pi_{j}^{\varepsilon_{n}}\right\rangle .
$$

Letting $n \rightarrow \infty$ we are led to a contradiction $\gamma \leq 0$.

If $r>0$ we proceed similarly. Namely, according to $(63)_{3}$,

$$
r^{\varepsilon_{n}} \gamma \leq r^{\varepsilon_{n}} \sum_{j=1}^{m} \phi_{j}\left(x^{\varepsilon_{n}}\right)+r^{\varepsilon_{n}} m \varepsilon_{n}=r^{\varepsilon_{n}} f_{0}\left(x^{\varepsilon_{n}}\right)+r^{\varepsilon_{n}} m \varepsilon_{n}=\left\langle x^{\varepsilon_{n}},-\sum_{j=1}^{m} \pi_{j}^{\varepsilon_{n}}\right\rangle .
$$

Passing to the limit as $n \rightarrow \infty$ yields again the contradiction $r \gamma \leq 0$.

Case $2 J_{\infty}=\emptyset$. In such a case our task is to show that $x \neq 0$ and $r>0$. As already established, $\pi_{j} \in \partial f_{j}\left(\alpha_{j} x\right)$ for each $j=1, \ldots, m$, and (65) holds.

If $r=0$ then it is easy to arrive at $\pi_{j}=0, j=1, \ldots, m$, which due to $\left(H_{10}^{\prime}\right)$ makes $x \neq 0$ and $\alpha_{j}>0$ for each $j=1, \ldots, m$. But this is only possible when $r=1$. The contradiction.

If $r>0$ and $x=0$ then we get $0 \in r \partial f_{0}(0)+\sum_{j=1}^{m} \partial f_{j}(0)$ which contradicts $\left(H_{7}^{\prime}\right)$ because $0 \in r \Delta^{\star}+\sum_{j=1}^{m} \partial f_{j}(0) \subset \Delta^{\star}+\sum_{j=1}^{m} \partial f_{j}(0)$.

The proof of Proposition 10 is complete. 
The assumption $\left(H_{4}\right)$ and $\left(H_{5}\right)$ seem to be too restrictive in many important infinite dimensional applications. Now we show that the equality $f_{0}(\cdot)=\sum_{j=1}^{m} \phi_{j}(\cdot)$ makes this assumption redundant. Recall that $\phi_{j}(\cdot)$ has been assumed to be convex, lower semicontinuous, positive homogeneous, nonnegative valued.

Now we are ready to formulate our main result concerning the economic equilibrium problem.

Theorem 6 Let $X$ be a reflexive Banach space and let $\mathcal{K}^{\star}$ be normal. Moreover, assume that for each $j \in\{1, \ldots, m\}$ the hypotheses below hold:

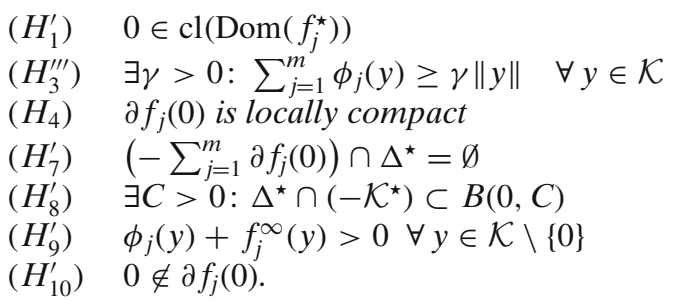

Then there exist $\left(x,\left(\pi_{j}\right),\left(\alpha_{j}\right)\right) \in \mathcal{K} \times\left(\mathcal{K}^{\star}\right)^{m} \times\left(\mathbb{R}_{+}\right)^{m},\|x\|=1$, and $r \in(0,1]$ such that

$$
\left.\begin{array}{c}
\pi_{j} \in \partial f_{j}\left(\alpha_{j} x\right), \quad j=1, \ldots, m \\
-\sum_{j=1}^{m} \pi_{j} \in r \partial f_{0}(x) \\
-\left\langle x, \pi_{j}\right\rangle-\phi_{j}(x) \in \partial \operatorname{ind}_{\geq 0}\left(\alpha_{j}\right), \quad j=1, \ldots, m,
\end{array}\right\}
$$

or equivalently, there exists $x \in \mathcal{K}, x \neq 0$ and $r \in(0,1]$ such that

$$
0 \in \mathcal{R}(x)+r \partial f_{0}(x) \text {. }
$$

Proof To show this result a method based on the finite intersection property will be used (cf. [12]). Let $\Theta$ be the family of all finite dimensional subspaces of $X$, ordered by inclusion. Let $F \in \Theta, i^{F}: F \rightarrow X$ be the injection of $F$ into $X$ and $i^{F \star}: X^{\star} \rightarrow F^{\star}$ be its adjoint. Set $f_{j}^{F}=f_{j} \circ i_{F}: F \rightarrow \overline{\mathbb{R}}, j=0,1, \ldots, m, \mathcal{K}^{F}=\left\{y \in X: i^{F}(y) \in \mathcal{K}\right\}=$ $\mathcal{K} \cap F, \phi_{j}^{F}=\phi_{j} \circ i^{F}: \mathcal{K}^{F} \rightarrow \mathbb{R}, j=1, \ldots, m$. Since $i^{F^{\star}}$ is surjective we get $\mathcal{K}^{F \star}=$ $i^{F \star}\left(\mathcal{K}^{\star}\right)$ where $\mathcal{K}^{F \star}$ is the negative polar of $\mathcal{K}^{F}$. Set $\Delta^{\star F}=i^{F \star}\left(\Delta^{\star}\right)$.

First we consider the case when $\left(H_{3}^{\prime \prime \prime}\right)$ is replaced by $\left(H_{3}^{\prime \prime}\right)$.

Notice that $\left(H_{4}\right)$ and $\left(H_{5}\right)$ are redundant in the setting of finite dimensional spaces. From (42) it follows that $\left\|\tau^{F}+\kappa^{F}\right\|_{F^{\star}} \geq \rho\left(\left\|\tau^{F}\right\|_{F^{\star}}+\left\|\kappa^{F}\right\|_{F^{\star}}\right) \forall \tau^{F}, \kappa^{F} \in \mathcal{K}^{F \star}$, $\|\cdot\|_{F^{\star}}$ being the norm in $F^{\star}$ defined by $\left\|i^{F^{\star}}(\tau)\right\|_{F^{\star}}=\inf _{\kappa \in \operatorname{Ker} i i^{*}}\|\tau+\kappa\|, \forall \tau \in X$. This allows to check that $\Delta^{\star F} \cap\left(-\mathcal{K}^{F \star}\right) \subset B_{F^{\star}}(0, C)$ with the same constant $C>0$ as in $\left(H_{8}^{\prime}\right)$ (not depending on $F$ ).

Further, set $F_{0} \in \Theta$ "large" enough to fulfill the conditions: $0 \notin \partial f_{j}^{F_{0}}(0), j=$ $1, \ldots, m$, and $i^{F_{0} \star}\left(\sum_{j=1}^{m} \partial f_{j}(0)+\Delta^{\star}\right) \neq 0$ which is possible due to $\left(H_{10}^{\prime}\right)$ and $\left(H_{7}^{\prime}\right)$, respectively.

Without loss of generality we can suppose that $\left.i^{F \star}\left(\partial f_{j}(0)\right)=\partial f_{j}^{F}(0)\right), j=1, \ldots, m$, $F \in \Theta$. Indeed, following the technique of Proposition 9 we can suppose that Dom $f_{j}^{\star}$, $j=1, \ldots m$, are bounded which implies that Dom $f_{j}=X, j=1, \ldots, m$, and the assertion follows. 
Now, if we denote by $\Theta_{F_{0}}$ the set of all finite dimensional subspaces of $X$ containing $F_{0}$ then for each $F \in \Theta_{F_{0}}$ all the assumptions of Proposition 9 are fulfilled. Consequently, the existence of $x^{F} \in F,\left\|x^{F}\right\|=1,\left(\pi_{j}^{F}\right) \in\left(\mathcal{K}^{\star}\right)^{m},\left(\alpha_{j}^{F}\right) \in \mathbb{R}_{+}^{m}$ and $r^{F} \in(0,1]$ such that

$$
\left.\begin{array}{c}
i^{F \star}\left(\pi_{j}^{F}\right) \in \partial f_{j}^{F}\left(\alpha_{j}^{F} x^{F}\right), \quad\left\langle x^{F}, \pi_{j}^{F}\right\rangle+\phi_{j}\left(x^{F}\right)=0, \quad \alpha_{j}^{F}>0 \\
i^{F \star}\left(\pi_{j}^{F}\right) \in \partial f_{j}^{F}(0), \quad\left\langle x^{F}, \pi_{j}^{F}\right\rangle+\phi_{j}\left(x^{F}\right) \geq 0, \quad \alpha_{j}^{F}=0 \\
-\sum_{j=1}^{m} i^{F \star}\left(\pi_{j}^{F}\right) \in r^{F} \partial f_{0}^{F}\left(x^{F}\right),
\end{array}\right\}
$$

follows. Hence,

$$
\left.\begin{array}{c}
f_{j}(y)-f_{j}\left(\alpha_{j}^{F} x^{F}\right) \geq\left\langle y-\alpha_{j}^{F} x^{F}, \pi_{j}^{F}\right\rangle \quad \forall y \in F, \quad \alpha_{j}^{F}>0 \\
f_{j}(y)-f_{j}(0) \geq\left\langle y, \pi_{j}^{F}\right\rangle \quad \forall y \in F, \quad \alpha_{j}^{F}=0 \\
f_{0}(y)-f_{0}\left(x^{F}\right) \geq\left\langle y-x^{F},-\frac{1}{r^{F}} \sum_{j=1}^{m} \pi_{j}^{F}\right\rangle \forall y \in F . \\
\left\langle x^{F}, \pi_{j}^{F}\right\rangle+\phi_{j}\left(x^{F}\right)=0, \quad \alpha_{j}^{F}>0 \\
\left\langle x^{F}, \pi_{j}^{F}\right\rangle+\phi_{j}\left(x^{F}\right) \geq 0, \quad \alpha_{j}^{F}=0 .
\end{array}\right\}
$$

By $\left(H_{8}^{\prime}\right)$ and (42) we can suppose that $\left\{\pi_{j}^{F}\right\}_{F \in \Theta_{F_{0}}}$ is bounded in $X^{\star}$. The boundedness of $\left\{\left(\alpha_{J}^{F}\right)\right\}_{F \in \Theta_{F_{0}}}$ follows from $\left(H_{3}^{\prime \prime}\right)$. Recall that $\left\{x^{F}\right\}_{F \in \Theta_{F_{0}}}$ lies on the unit sphere and $r^{F} \in(0,1]$. Therefore

$$
\left.\Omega_{F}=\bigcup_{\substack{F^{\prime} \in \Theta_{F_{0}} \\ F^{\prime} \supset F}}\left\{\left(x^{F^{\prime}},\left(\pi_{j}^{F^{\prime}}\right),\left(\alpha_{j}^{F^{\prime}}\right), r^{F^{\prime}}\right)\right)\right\}, \quad F \in \Theta_{F_{0}}
$$

is contained in the closed ball $B_{X \times\left(X^{\star}\right)^{m} \times \mathbb{R}_{+}^{m} \times \mathbb{R}}(0, R)$ of $X \times\left(X^{\star}\right)^{m} \times \mathbb{R}_{+}^{m} \times \mathbb{R}$ with the radius $R$ not depending on $F \in \Theta_{F_{0}}$. Since $B_{X \times\left(X^{\star}\right)^{m} \times \mathbb{R}_{+}^{m} \times \mathbb{R}}(0, R)$ is weakly compact and the family $\left\{\text { weakcl }\left(\Omega_{F}\right)\right\}_{F \in \Theta_{F_{0}}}$ has the finite intersection property (easy to be checked), the intersection

$$
\bigcap_{F \in \Theta_{F_{0}}}\left\{\operatorname{weakcl}\left(\Omega_{F}\right)\right\} \neq \emptyset
$$

is not empty, say

$$
\left(x,\left(\pi_{j}\right),\left(\alpha_{j}\right), r\right) \in \bigcap_{F \in \Theta_{F_{0}}}\left\{\operatorname{weakcl}\left(\Omega_{F}\right)\right\} .
$$

Let $z \in X$ be arbitrarily given. Choose $F \in \Theta_{F_{0}}$ containing $x$ and $z$. Taking into account (69) we are allowed to extract a sequence $\left(x^{F_{n}},\left(\pi_{j}^{F_{n}}\right),\left(\alpha_{j}^{F_{n}}\right), r^{F_{n}}\right)$ in $\Omega_{F}$ such that $x^{F_{n}} \rightarrow x$ weakly in $X, \pi_{j}^{F_{n}} \rightarrow \pi_{j}$ weakly in $X^{\star},\left(\alpha_{j}^{F_{n}}\right) \rightarrow\left(\alpha_{j}\right)$ in $\mathbb{R}^{m}$ and $r^{F_{n}} \rightarrow r$ in $\mathbb{R}$ as $n \rightarrow \infty$.

By substituting $y \mapsto x$ into (68) we show by the way already presented that

$$
\lim _{n \rightarrow \infty}\left\langle x^{F_{n}}, \pi_{j}^{F_{n}}\right\rangle=\left\langle x, \pi_{j}\right\rangle, \quad j=1, \ldots, m .
$$


Now letting $n \rightarrow \infty$ in (68) leads to

$$
\left.\begin{array}{c}
f_{j}(z)-f_{j}\left(\alpha_{j} x\right) \geq\left\langle z-\alpha_{j} x, \pi_{j}\right\rangle, \quad \alpha_{j}>0 \\
f_{j}(z)-f_{j}(0) \geq\left\langle z, \pi_{j}\right\rangle, \quad \alpha_{j}=0 \\
r f_{0}(z)-r f_{0}(x) \geq\left\langle z-x,-\sum_{j=1}^{m} \pi_{j}\right\rangle .
\end{array}\right\}
$$

Since $z \in X$ has been chosen arbitrarily,

$$
\begin{aligned}
& f_{j}(z)-f_{j}\left(\alpha_{j} x\right) \geq\left\langle z-\alpha_{j} x, \pi_{j}\right\rangle, \quad \forall z \in \mathcal{K} . \\
& r f_{0}(z)-r f_{0}(x) \geq\left\langle z-x,-\sum_{j=1}^{m} \pi_{j}\right\rangle, \quad \forall z \in \mathcal{K},
\end{aligned}
$$

i.e. $\pi_{j} \in \partial f_{j}\left(\alpha_{j} x\right), j=1, \ldots, m$, and $-\sum_{j=1}^{m} \pi_{j} \in r \partial f_{0}(x)$.

We claim that $x \neq 0$ and $r>0$.

Indeed, if $r=0$ then from $(72)_{2}$ it follows that $-\sum_{j=1}^{m} \pi_{j} \in \mathcal{K}^{\star}$. On the other hand, $\sum_{j=1}^{m} \pi_{j} \in \mathcal{K}^{\star}$ which in view of $\mathcal{K}^{\star} \cap\left(-\mathcal{K}^{\star}\right)=\{0\}$ yields $\sum_{j=1}^{m} \pi_{j}=0$ and consequently, $\pi_{j}=0$ for each $j=1, \ldots, m\left(\mathcal{K}^{\star}\right.$ is normal). Thus $0 \in \partial f_{j}\left(\alpha_{j} x\right), j=1, \ldots, m$, which by $\left(H_{10}\right)$ means that $x \neq 0$ and $\alpha_{j}>0$ for each $j=1, \ldots, m$. Further on, from $(68)_{3}$ we get

$$
0<\gamma_{j} \leq \phi_{j}\left(x^{F_{n}}\right)=-\left\langle x^{F_{n}}, \pi_{j}^{F_{n}}\right\rangle
$$

which by passing to the limit leads to the contradiction $\gamma_{j} \leq 0$. This contradiction ensures that $r>0$.

Let $x=0$. According to $(72)_{2}, f_{0}(z) \geq\left\langle z,-\frac{1}{r} \sum_{j=1}^{m} \pi_{j}\right\rangle$ for all $z \in \mathcal{K}$, which is equivalent to $-\frac{1}{r} \sum_{j=1}^{m} \pi_{j} \in \Delta^{\star}$. But $0 \in \Delta^{\star}$, so $-\sum_{j=1}^{m} \partial f_{j}(0) \ni-\sum_{j=1}^{m} \pi_{j} \in r \Delta^{\star} \subset \Delta^{\star}$ in the contrary to $\left(H_{7}^{\prime}\right)$. The assertion follows.

Since $f_{0}$ is weakly lower semicontinuous, $\liminf _{n \rightarrow \infty} f_{0}\left(x^{F_{n}}\right) \geq f_{0}(x)$. But from $(68)_{3}$ and (70) it follows that $\limsup _{n \rightarrow \infty} f_{0}\left(x^{F_{n}}\right) \leq f_{0}(x)$ which implies

$$
\lim _{n \rightarrow \infty} f_{0}\left(x^{F_{n}}\right)=f_{0}(x)
$$

Taking into account that $f_{0}(\cdot)=\sum_{j=1}^{m} \phi_{j}(\cdot)$ and that each of $\phi_{j}(\cdot)$ 's is weakly lower semicontinuous, we obtain $\lim _{n \rightarrow \infty} \phi_{j}\left(x^{F_{n}}\right)=\phi_{j}(x), j=1, \ldots, m$. Having in mind (70), thanks to $(68)_{4-5}$ we are led to $(62)_{3}$. Summing up, with the use of $\left(H_{3}^{\prime \prime}\right)$ we have established (62). It must be emphasized that $\left(H_{5}\right)$ has not been applied in the aforementioned procedure.

In the last step we return to $\left(H_{3}^{\prime \prime \prime}\right)$. The idea is to consider the regularization $\phi_{j}(\cdot)+\varepsilon\|\cdot\|, \varepsilon>0$, instead of $\phi_{j}(\cdot)$. Then for sufficiently small $\varepsilon>0$ the existence of $\left(x^{\varepsilon},\left(\pi_{j}^{\varepsilon}\right),\left(\alpha_{j}^{\varepsilon}\right), r^{\varepsilon}\right),\left\|x^{\varepsilon}\right\|=1$, follows such that

$$
\left.\begin{array}{c}
\pi_{j}^{\varepsilon} \in \partial f_{j}\left(\alpha_{j}^{\varepsilon} x\right), \quad j=1, \ldots, m \\
-\sum_{j=1}^{m} \pi_{j}^{\varepsilon} \in r^{\varepsilon} \partial f_{0}^{\varepsilon}\left(x^{\varepsilon}\right) \\
-\left\langle x^{\varepsilon}, \pi_{j}^{\varepsilon}\right\rangle-\phi_{j}\left(x^{\varepsilon}\right)-\varepsilon\left\|x^{\varepsilon}\right\| \in \partial \operatorname{ind}_{\geq 0}\left(\alpha_{j}^{\varepsilon}\right), \quad j=1, \ldots, m,
\end{array}\right\}
$$


where $f_{0}^{\varepsilon}(\cdot)=f_{0}(\cdot)+\varepsilon m\|\cdot\|$. By the boundedness arguments we can assume that for a sequence $\varepsilon_{n} \rightarrow 0$ as $n \rightarrow \infty, x^{\varepsilon_{n}} \rightarrow x$ and $\pi_{j}^{\varepsilon_{n}} \rightarrow \pi_{j}$ weakly in $X$ and $X^{\star}$, respectively, and $r^{\varepsilon_{n}} \rightarrow r$. On the way already presented we arrive at $\lim _{n \rightarrow \infty}\left\langle x^{\varepsilon_{n}}, \pi_{j}^{\varepsilon_{n}}\right\rangle=$ $\left\langle x, \pi_{j}\right\rangle, j=1, \ldots, m$. Thus $\pi_{j} \in \partial f_{j}\left(\alpha_{j} x\right)$ provided that $j \in J=\left\{i \in\{1, \ldots, m\}: \alpha_{i}^{\varepsilon_{n}} \rightarrow\right.$ $\left.\alpha_{i} \in \mathbb{R}_{+}\right\}$. If $j \in J_{\infty}=\left\{i \in\{1, \ldots, m\}: \alpha_{i}^{\varepsilon_{n}} \rightarrow+\infty\right\}$ then from

$$
0 \geq-\varepsilon_{n}\left\|x^{\varepsilon_{n}}\right\|=\phi_{j}\left(x^{\varepsilon_{n}}\right)+\frac{1}{\alpha_{j}^{\varepsilon_{n}}} f_{j}\left(\alpha_{j}^{\varepsilon} x^{\varepsilon_{n}}\right)+\frac{1}{\alpha_{j}^{\varepsilon_{n}}} f_{j}^{\star}\left(\pi_{j}^{\varepsilon_{n}}\right), \quad j=1, \ldots, m,
$$

it results

$$
0 \geq \phi_{j}(x)+f_{j}^{\infty}(x)
$$

Moreover, passing to the limit in $(73)_{3}$ yields

$$
r f_{0}(y)-r f_{0}(x) \geq\left\langle y-x,-\sum_{j=1}^{m} \pi_{j}\right\rangle \quad \forall y \in \mathcal{K} .
$$

Proceeding analogously as in the proof of Proposition 10 we establish $(62)_{1-2}$ with $x \neq 0$ and $r>0$.

It remains to confirm $(62)_{3}$. Since $f_{0}$ is weakly lower semicontinuous,

$$
\liminf _{n \rightarrow \infty} f_{0}\left(x^{\varepsilon_{n}}\right) \geq f_{0}(x)
$$

But from (73) 3 and (70) it follows that $\lim _{\sup _{n \rightarrow \infty}} f_{0}\left(x^{\varepsilon_{n}}\right) \leq f_{0}(x)$ which implies

$$
\lim _{n \rightarrow \infty} f_{0}\left(x^{\varepsilon_{n}}\right)=f_{0}(x)
$$

Taking into account that $f_{0}(\cdot)=\sum_{j=1}^{m} \phi_{j}(\cdot)$ and that each of $\phi_{j}(\cdot)$ 's is weakly lower semicontinuous, we obtain

$$
\lim _{n \rightarrow \infty} \phi_{j}\left(x^{\varepsilon_{n}}\right)=\phi_{j}(x), \quad j=1, \ldots, m
$$

Having in mind (70), thanks to $(73)_{3}$ we are led to $(62)_{3}$. This completes the proof of Theorem 6.

Corollary 1 Under the assumptions of Theorem $6,\left(x,\left(\pi_{j}\right), r\right) \in \mathcal{K} \times\left(\mathcal{K}^{\star}\right)^{m} \times(0,1]$ is a solution of the following economic equilibrium problem:

Find $\pi_{j} \in \mathcal{K}^{\star}, j=1, \ldots, m$, and $x \in \mathcal{K},\|x\|=1$, such that

$$
\left.\begin{array}{l}
f_{j}^{\star}\left(\pi_{j}\right)=\inf \left\{f_{j}^{\star}(\tau):-\langle x, \tau\rangle \leq \phi_{j}(x) \text { and } \tau \in \mathcal{K}^{\star}\right\}, \quad j=1, \ldots, m \\
\sum_{j=1}^{m} \phi_{j}(x)=-\frac{1}{r} \sum_{j=1}^{m}\left\langle x, \pi_{j}\right\rangle .
\end{array}\right\}
$$

If for each $j=1, \ldots, m$ the inequality constraints $-\langle x, \tau\rangle \leq \phi_{j}(x)$ hold as equalities then $r=1$. 
In the case $\partial f_{j}(0)=\emptyset, j=1, \ldots, m$, from Theorem 6 we get the result.

Corollary 2 Let $\mathcal{K}^{\star}$ be normal. Moreover, assume that for each $j \in\{1, \ldots, m\}$ the hypotheses below hold:

$\left(H_{1}^{\prime}\right) \quad 0 \in \operatorname{cl}\left(\operatorname{Dom}\left(f_{j}^{\star}\right)\right)$

$\left(H_{3}^{\prime \prime \prime}\right) \quad \exists \gamma>0: \sum_{j=1}^{m} \phi_{j}(y) \geq \gamma\|y\| \quad \forall y \in \mathcal{K}$

$\left(H_{8}^{\prime}\right) \quad \exists C>0: \Delta^{\star} \cap\left(-\mathcal{K}^{\star}\right) \subset B(0, C)$

$\left(H_{9}^{\prime}\right) \quad \phi_{j}(y)+f_{j}^{\infty}(y)>0 \forall y \in \mathcal{K} \backslash\{0\}$

$\left(H_{10}^{\prime}\right) \quad \partial f_{j}(0)=\emptyset$,

there exist $\left(x,\left(\pi_{j}\right),\left(\alpha_{j}\right)\right) \in \mathcal{K} \times\left(\mathcal{K}^{\star}\right)^{m} \times \operatorname{Int}\left(\mathbb{R}_{+}^{m}\right)$, $\|x\|=1$, such that

$$
\left.\begin{array}{c}
\pi_{j} \in \partial f_{j}\left(\alpha_{j} x\right), \quad j=1, \ldots, m \\
-\sum_{j=1}^{m} \pi_{j} \in \partial f_{0}(x) \\
\left\langle x, \pi_{j}\right\rangle+\phi_{j}(x)=0, \quad j=1, \ldots, m .
\end{array}\right\}
$$

Moreover, $\pi_{j} \in \mathcal{K}^{\star}, j=1, \ldots, m$, and $x \in \mathcal{K},\|x\|=1$, are solutions of the problem

$$
\left.\begin{array}{l}
f_{j}^{\star}\left(\pi_{j}\right)=\inf \left\{f_{j}^{\star}(\tau):-\langle x, \tau\rangle \leq \phi_{j}(x) \text { and } \tau \in \mathcal{K}^{\star}\right\}, \quad j=1, \ldots, m \\
\sum_{j=1}^{m} \phi_{j}(x)=-\sum_{j=1}^{m}\left\langle x, \pi_{j}\right\rangle .
\end{array}\right\}
$$

If $\left(H_{3}^{\prime \prime \prime}\right)$ is replaced by $\left(H_{3}^{\prime \prime}\right)$ then $\left(H_{9}^{\prime}\right)$ is redundant and the following result follows.

Corollary 3 Let $\mathcal{K}^{\star}$ be normal. Moreover, assume that for each $j \in\{1, \ldots, m\}$ the hypotheses below hold:

$\left(H_{1}^{\prime}\right) \quad 0 \in \operatorname{cl}\left(\operatorname{Dom}\left(f_{j}^{\star}\right)\right)$

$\left(H_{3}^{\prime \prime}\right) \quad \exists \gamma_{j}>0: \phi_{j}(y) \geq \gamma_{j}\|y\| \quad \forall y \in \mathcal{K}$

$\left(H_{8}^{\prime}\right) \quad \exists C>0: \Delta^{\star} \cap\left(-\mathcal{K}^{\star}\right) \subset B(0, C)$

$\left(H_{10}^{\prime}\right) \quad \partial f_{j}(0)=\emptyset$,

there exist $\left(x,\left(\pi_{j}\right),\left(\alpha_{j}\right)\right) \in \mathcal{K} \times\left(\mathcal{K}^{\star}\right)^{m} \times \operatorname{Int}\left(\mathbb{R}_{+}^{m}\right),\|x\|=1$, such that (76) holds.

Remark 4 In Theorem 6, if $\left(H_{9}^{\prime}\right)$ does not hold for some $j \in\{1, \ldots, m\}$ then there might happen that $\phi_{j}(x)=-\left\langle x, \pi_{j}\right\rangle=0$ and $\alpha_{j}=+\infty$ (cf. [34]). This is a warning signal for $j$ 's consumer that the bankruptcy may occur.

\section{Constrained Multiobjective Optimization Problem}

In this section some existence results for a class of multiobjective optimization problems with "budget-like" constraints will be established. For any $\beta=\left(\beta_{j}\right) \in \operatorname{Int} \mathbb{R}^{m}$ define

$$
f_{j}^{\beta}(y)=f_{j}\left(\frac{1}{\beta_{j}} y\right), \quad y \in \mathcal{K}, \quad j=1, \ldots, m .
$$


It is easy to see that

$$
\pi \in \partial f_{j}^{\beta}(x) \Leftrightarrow \beta_{j} \pi \in \partial f_{j}\left(\frac{1}{\beta_{j}} x\right) .
$$

One can check immediately that if $f_{0}, f_{j}, \phi_{j}$ fulfill the assumptions of Corollary 3 then for the data system $\left(f_{0}, f_{j}^{\beta}, \phi_{j}\right)$ all these assumptions are fulfilled as well. Accordingly, the result follows.

Proposition 11 Assume that $\mathcal{K}^{\star}$ is normal. Moreover, suppose that for each $j=$ $1, \ldots, m$, the hypotheses below hold:

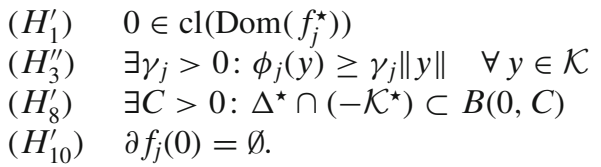

Then for each $\beta \in\left(\operatorname{Int} \mathbb{R}_{+}\right)^{m}$ there exists $\left(x^{\beta},\left(\pi_{j}^{\beta}\right),\left(\alpha_{j}^{\beta}\right)\right) \in\left(\mathcal{K} \backslash\{0\} \times\left(\mathcal{K}^{\star}\right)^{m} \times\left(\mathbb{R}_{+}\right)^{m}\right)$ such that

$$
\left.\begin{array}{c}
\pi_{j}^{\beta} \in \frac{1}{\beta_{j}} \partial f_{j}\left(\frac{\alpha_{j}^{\beta}}{\beta_{j}} x^{\beta}\right) \\
-\sum_{j=1}^{m} \pi_{j}^{\beta} \in \partial f_{0}\left(x^{\beta}\right) \\
-\left\langle x^{\beta}, \pi_{j}^{\beta}\right\rangle-\phi_{j}\left(x^{\beta}\right)=0
\end{array}\right\} \quad j=1, \ldots, m .
$$

Corollary 4 Under the assumptions of Proposition 11 it follows

$$
\left.\begin{array}{c}
\sum_{j=1}^{m} \frac{1}{\alpha_{j}^{\beta}} f_{j}\left(\frac{\alpha_{j}^{\beta}}{\beta_{j}} x^{\beta}\right)+f_{0}\left(x^{\beta}\right)+\sum_{j=1}^{m} \frac{1}{\alpha_{j}^{\beta}} f_{j}^{\star}\left(\beta_{j} \pi_{j}^{\beta}\right)=0 \\
\sum_{j=1}^{m} \frac{1}{\alpha_{j}^{\beta}} f_{j}\left(\frac{\alpha_{j}^{\beta}}{\beta_{j}} x^{\beta}\right)+f_{0}\left(x^{\beta}\right) \leq \sum_{j=1}^{m} \frac{1}{\alpha_{j}^{\beta}} f_{j}\left(\frac{\alpha_{j}^{\beta}}{\beta_{j}} y\right)+f_{0}(y) \quad \forall y \in \mathcal{K} \\
\sum_{j=1}^{m} \frac{1}{\alpha_{j}^{\beta}} f_{j}^{\star}\left(\beta_{j} \pi_{j}^{\beta}\right) \leq \sum_{j=1}^{m} \frac{1}{\alpha_{j}^{\beta}} f_{j}^{\star}\left(\beta_{j} \tau_{j}\right), \forall\left(\tau_{j}\right) \in\left(\mathcal{K}^{\star}\right)^{m},-\sum_{j=1}^{m} \tau_{j} \in \Delta^{\star} .
\end{array}\right\}
$$

Define a mapping $\Gamma: \operatorname{Int}\left(\mathbb{R}_{+}^{m}\right) \rightarrow 2^{\mathbb{R}^{m}}$ by assigning to each $\beta \in \operatorname{Int}\left(\mathbb{R}_{+}^{m}\right)$ the set of all corresponding Lagrange multipliers, i.e.

$$
\begin{aligned}
\Gamma(\beta):= & \left\{\left(\alpha_{j}^{\beta}\right) \in \mathbb{R}_{+}^{m}:\left(\left(x^{\beta},\left(\pi_{j}^{\beta}\right),\left(\alpha_{j}^{\beta}\right)\right) \text { is a solution of }(77)\right.\right. \\
& \text { for some } \left.\left(x^{\beta},\left(\pi_{j}^{\beta}\right)\right) \in\left(\mathcal{K} \times\left(\mathcal{K}^{\star}\right)^{m}\right) \text { with } f_{0}\left(x^{\beta}\right)=c\right\} .
\end{aligned}
$$

Here $c$ is a positive constant which can be arbitrarily specified thanks to the positive homogeneity of $\phi_{j}$ and $f_{0}=\sum_{j=1}^{m} \phi_{j}$. Note that $\Gamma(\cdot)$ has nonempty, compact values, but it is not convex valued. 
Our aim now is to examine the conditions under which $\Gamma$ has at least one fixed point. Notice, that if $\Gamma$ happens to have a fixed point, say $\beta$, the corresponding $x^{\beta}$ is a solution of the minimizing problem

$$
\sum_{j=1}^{m} \frac{1}{\beta_{j}} f_{j}\left(x^{\beta}\right)+f_{0}\left(x^{\beta}\right) \leq \sum_{j=1}^{m} \frac{1}{\beta_{j}} f_{j}(y)+f_{0}(y) \quad \forall y \in \mathcal{K}
$$

with the "budget-like" constraints

$$
-\left\langle x^{\beta}, \pi_{j}^{\beta}\right\rangle-\phi_{j}\left(x^{\beta}\right)=0, \quad j=1, \ldots, m
$$

It is easy to observe that in such a case $x^{\beta}$ is a Pareto optimal solution of a multiobjective optimization problem related to the vector objective $\left(f_{0}, f_{1}, \ldots, f_{m}\right)$. Moreover, the validity of "budget-like" constraints (81) is ensured.

Proposition 12 Under the assumptions of Proposition 11 and

$\left(H_{11}\right) \quad \partial f_{j}^{\star}(0)=\emptyset$,

there exist $\sigma, S \in \operatorname{Int} \mathbb{R}_{+}^{m}$ with $\sigma_{j}<S_{j}, j=1, \ldots, m$, such that

$$
\beta=\left(\beta_{j}\right) \in[\sigma, S] \Longrightarrow\left(\alpha_{j}^{\beta}\right) \in[\sigma, S],
$$

where $[\sigma, S]=\left\{\left(t_{j}\right): \sigma_{j} \leq t_{j} \leq S_{j}, j=1, \ldots, m\right\}$, i.e. $\Gamma([\sigma, S]) \subset[\sigma, S]$.

Proof Let $\beta \in \operatorname{Int} \mathbb{R}_{+}^{m}$ and

$$
\left(x^{\beta},\left(\pi_{j}^{\beta}\right),\left(\alpha_{j}^{\beta}\right)\right), \quad x^{\beta} \neq 0,
$$

be a solution of (77). Since $f_{0}, \phi_{j}$ are positive homogeneous, one can suppose that

$$
\left\|x^{\beta}\right\|=c>0
$$

for some fixed $c>0$ which will be specified later.

Since $f_{j}^{\star}$ is convex, proper and lower semicontinuous, there exist $a_{j}, b_{j} \geq 0$ with

$$
f_{j}^{\star}(\tau) \geq-a_{j}-b_{j}\|\tau\|, \quad \forall \tau \in \operatorname{Dom}\left(f_{j}^{\star}\right) .
$$

In view of $\left(H_{8}^{\prime}\right)$ and $(42)$, from $(77)_{2}$ it follows

$$
\left\|\pi_{j}^{\beta}\right\| \leq \frac{C}{\mu^{m-1}}
$$

Now we claim that for each $S=\left(S_{j}\right) \in \operatorname{Int} \mathbb{R}_{+}^{m}$ there exists $\sigma_{S}=\left(\sigma_{S j}\right), \sigma_{S j}>0$, such that

$$
\beta=\left(\beta_{j}\right) \in\left[\sigma_{S}, S\right] \Longrightarrow\left(\alpha_{j}^{\beta}\right) \in\left[\sigma_{S}, \infty\right) .
$$


On the contrary, suppose that for some $j \in\{1, \ldots, m\}$ there exists $\sigma_{j}^{n} \rightarrow 0$ as $n \rightarrow \infty$, such that $\sigma_{j}^{n} \leq \beta_{j}^{n} \leq S_{j}$ and $\alpha_{j}^{\beta^{n}}<\sigma_{j}^{n}$. Thus $\alpha_{j}^{\beta^{n}} \rightarrow 0$ as $n \rightarrow \infty$ and

$$
\frac{\alpha_{j}^{\beta^{n}}}{\beta_{j}^{n}} \leq \frac{\alpha_{j}^{\beta^{n}}}{\sigma_{j}^{n}}<1 .
$$

One can suppose that

$$
\frac{\alpha_{j}^{\beta^{n}}}{\beta_{j}^{n}} \rightarrow r, \quad \text { as } n \rightarrow \infty, \quad r \in[0,1] .
$$

Now consider two possible cases: 1) $r=0$ and 2) $r \in(0,1]$.

1) If $r=0$, then

$$
\frac{\alpha_{j}^{\beta^{n}}}{\beta_{j}^{n}} x^{\beta^{n}} \rightarrow 0, \quad \text { strongly in } X .
$$

By the boundedness of $\left(\beta_{j}^{n}\right)$ we are allowed to suppose that $\beta_{j}^{n} \pi_{j}^{n} \rightarrow \hat{\pi}_{j}$ weakly in $X^{\star}$ for some $\hat{\pi}_{j} \in X^{\star}$. Since $\beta_{j}^{n} \pi_{j}^{\beta^{n}} \in \partial f_{j}\left(\frac{\alpha_{j}^{\beta^{n}}}{\beta_{j}^{n}} x^{\beta^{n}}\right)$, thanks to the maximal monotonicity of $\partial f_{j}$ it follows that $\hat{\pi}_{j} \in \partial f_{j}(0)$, in contrary to $\left(H_{10}^{\prime}\right)$.

2) If $r \in(0,1]$, then $\beta_{j}^{n} \rightarrow 0$. By the boundedness of the corresponding $x^{\beta^{n}}$ and $\pi_{j}^{\beta^{n}}$ it follows that $\beta_{j}^{n} \pi_{j}^{\beta^{n}} \rightarrow 0$ strongly in $X^{\star}$ and $\frac{\alpha_{j}^{\beta^{n}}}{\beta_{j}^{n}} x^{\beta^{n}} \rightarrow r x$ weakly in $X$ for some $x \in X$ (passing to a subsequence, if necessary). Since $\frac{\alpha_{j}^{\beta^{n}}}{\beta_{j}^{n}} x^{\beta^{n}} \in \partial f_{j}^{\star}\left(\beta_{j}^{n} \pi_{j}^{\beta^{n}}\right)$, thanks to the maximal monotonicity of $\partial f_{j}^{\star}$ it follows that $r x \in \partial f_{j}^{\star}(0)$, in contrary to $\left(H_{11}\right)$. The claim has been established.

From now on, fix $S_{j}=S, j=1, \ldots, m$, with $S \geq 2$. For simplicity of notations we use the same symbol $S$ for the vector $(S, \ldots, S)$. As already established, there exists $\sigma_{S}$ corresponding to $S$ such that

$$
\beta_{j} \in\left[\sigma_{S j}, S\right] \Longrightarrow \alpha_{j}^{\beta} \in\left[\sigma_{S j}, \infty\right), \quad j=1, \ldots, m
$$

Using $(77)_{1}$ we get

$$
\begin{aligned}
\alpha_{j}^{\beta} \hat{\gamma} c & \leq \alpha_{j}^{\beta} \phi_{j}\left(x^{\beta}\right)=-\alpha_{j}^{\beta}\left\langle x^{\beta}, \pi_{j}^{\beta}\right\rangle \leq-\alpha_{j}^{\beta}\left\langle x^{\beta}, \tau\right\rangle+f_{j}^{\star}\left(\beta_{j} \tau\right)-f_{j}^{\star}\left(\beta_{j} \pi_{j}^{\beta}\right) \\
& \leq \alpha_{j}^{\beta}\left\|x^{\beta}\right\|\|\tau\|+f_{j}^{\star}\left(\beta_{j} \tau\right)+a_{j}+b_{j} \beta_{j}\left\|\pi_{j}^{\beta}\right\| \\
& \leq \alpha_{j}^{\beta} c\|\tau\|+f_{j}^{\star}\left(\beta_{j} \tau\right)+a_{j}+b_{j} \beta_{j} \frac{C}{\mu^{m-1}},
\end{aligned}
$$

$\hat{\gamma}=\min _{j=1, \ldots, m}\left\{\gamma_{j}\right\}$, which is valid for any $\tau \in \mathcal{K}^{\star}$ and $\beta \in \operatorname{Int} \mathbb{R}_{+}^{m}$. Substituting $\tau \in \mathcal{K}^{\star}$ with $\|\tau\| \leq \frac{\hat{\gamma}}{2}$ yields

$$
\alpha_{j}^{\beta} \frac{\hat{\gamma}}{2} c \leq f_{j}^{\star}\left(\beta_{j} \tau\right)+a_{j}+b_{j} \beta_{j} \frac{C}{\mu^{m-1}}
$$


Now define

$$
d_{S j}:=\max \left\{0, \inf \left\{f_{j}^{\star}\left(t_{j} \tau\right):\|\tau\| \leq \frac{\hat{\gamma}}{2}, \sigma_{S j} \leq t_{j}\right\}\right\} .
$$

Then the following estimate follows

$$
\alpha_{j}^{\beta} \frac{\hat{\gamma}}{2} c \leq d_{S j}+a_{j}+b_{j} \beta_{j} \frac{C}{\mu^{m-1}}, \quad \forall \beta_{j} \in\left[\sigma_{S j}, S\right]
$$

from which we obtain

$$
\begin{aligned}
\sum_{j=1}^{m} \alpha_{j}^{\beta} & \leq \frac{2}{c \hat{\gamma}} \sum_{j=1}^{m}\left(d_{S j}+a_{j}+b_{j} \beta_{j} \frac{C}{\mu^{m-1}}\right) \\
& \leq \frac{2}{c \hat{\gamma}} \sum_{j=1}^{m}\left(d_{S j}+a_{j}\right)+S \frac{2 C}{c \hat{\gamma} \mu^{m-1}} \sum_{j=1}^{m} b_{j} \quad \forall \beta \in\left[\sigma_{S}, S\right] .
\end{aligned}
$$

Now we are ready to choose $c>0$ in such a way to fulfill the desired condition

$$
\beta \in\left[\sigma_{S}, S\right] \Longrightarrow \alpha^{\beta} \in\left[\sigma_{S}, S\right] .
$$

Namely, if

$$
\max \left\{\frac{2}{\hat{\gamma}} \sum_{j=1}^{m}\left(d_{S j}+a_{j}\right), \frac{4 C}{\hat{\gamma} \mu^{m-1}} \sum_{j=1}^{m} b_{j}\right\} \leq c
$$

then

$$
\sum_{j=1}^{m} \alpha_{j}^{\beta} \leq 1+\frac{1}{2} S \leq S
$$

because $S \geq 2$. The proof of Proposition 12 is complete.

Proposition 13 Under the assumptions of Proposition 12 the mapping $\Gamma$ has at least one fixed point.

Proof By (77) and $(78)_{1}$, and the boundedness of $\left(x^{\beta},\left(\pi_{j}^{\beta}\right)\right)$ in $X \times\left(X^{\star}\right)^{m}$ we conclude that $\Gamma$ has compact, nonempty values and it is closed in the sense that whenever $\beta^{n} \rightarrow \beta$ as $n \rightarrow \infty$ then each cluster point of $\left\{\alpha^{\beta^{n}}\right\} \subset \Lambda\left(\beta^{n}\right)$ lies in $\Lambda(\beta)$. Therefore, a function $\Psi:[\sigma, S] \times[\sigma, S] \rightarrow \mathbb{R}$ given by

$$
\Psi(\beta, \alpha)=\min _{\tilde{\alpha} \in \Lambda(\beta)}\langle\beta-\widetilde{\alpha}, \beta-\alpha\rangle_{\mathbb{R}^{m}}, \quad(\beta, \alpha) \in[\sigma, S] \times[\sigma, S],
$$

where $\langle\cdot, \cdot\rangle_{\mathbb{R}^{m}}$ stands for the inner product in $\mathbb{R}^{m}$, is concave with respect to $\alpha$ (fixed $\beta$ ) and lower semicontinuous as a function of $\beta$ (fixed $\alpha$ ). By the Ky Fan inequality [8] there exists $\hat{\beta} \in[\sigma, S]$ with the property that

$$
\Psi(\hat{\beta}, \alpha) \leq \Psi(\alpha, \alpha)=0, \quad \forall \alpha \in[\sigma, S],
$$

This implies the existence of $\alpha^{\hat{\beta}} \in \Lambda(\hat{\beta})$ such that $\alpha^{\hat{\beta}}=\hat{\beta}$, i.e. $\hat{\beta} \in\left[\sigma_{S}, S\right]$ is a fixed point of $\Lambda$. The proof is complete. 
Proposition 14 Under the assumptions of Proposition 12 there exists $\left(x^{\beta},\left(\pi_{j}^{\beta}\right),\left(\beta_{j}\right)\right)$ such that

$$
\left.\begin{array}{c}
\pi_{j}^{\beta} \in \frac{1}{\beta_{j}} \partial f_{j}\left(x^{\beta}\right) \\
-\sum_{j=1}^{m} \pi_{j}^{\beta} \in \partial f_{0}\left(x^{\beta}\right) \\
-\left\langle x^{\beta}, \pi_{j}^{\beta}\right\rangle-\phi_{j}\left(x^{\beta}\right)=0
\end{array}\right\} \quad j=1, \ldots, m .
$$

Moreover,

$$
\left.\begin{array}{c}
\sum_{j=1}^{m} \frac{1}{\beta_{j}} f_{j}\left(x^{\beta}\right)+f_{0}\left(x^{\beta}\right)+\sum_{j=1}^{m} \frac{1}{\beta_{j}} f_{j}^{\star}\left(\beta_{j} \pi_{j}^{\beta}\right)=0 \\
\sum_{j=1}^{m} \frac{1}{\beta_{j}} f_{j}\left(x^{\beta}\right)+f_{0}\left(x^{\beta}\right) \leq \sum_{j=1}^{m} \frac{1}{\beta_{j}} f_{j}(y)+f_{0}(y) \quad \forall y \in \mathcal{K} \\
\sum_{j=1}^{m} \frac{1}{\beta_{j}} f_{j}^{\star}\left(\beta_{j} \pi_{j}^{\beta}\right) \leq \sum_{j=1}^{m} \frac{1}{\beta_{j}} f_{j}^{\star}\left(\beta_{j} \tau_{j}\right), \quad \forall\left(\tau_{j}\right) \in\left(\mathcal{K}^{\star}\right)^{m},-\sum_{j=1}^{m} \tau_{j} \in \Delta^{\star} .
\end{array}\right\}
$$

In other words, a multiobjective optimization problem with "budget-like" constraints (related to the vector objective $\left(f_{0}, f_{1}, \ldots, f_{m}\right)$ and the "budget functions" $\left.\left(\phi_{1}, \ldots, \phi_{m}\right)\right)$ admits at least one Pareto optimal solution.

Now we are ready to formulate the main result of this chapter.

Theorem 7 Assume that $\mathcal{K}^{\star}$ is normal and $f_{0}(y)=\sum_{j=1}^{m} \phi_{j}(y), \quad y \in \mathcal{K}$, where $\phi_{j}(\cdot)$ are convex, lower semicontinuous, positive homogeneous, nonnegative valued. Suppose that for each $j=1, \ldots$, m the hypotheses below hold:

$\left(H_{1}^{\prime}\right) \quad 0 \in \operatorname{cl}\left(\operatorname{Dom}\left(f_{j}^{\star}\right)\right)$

$\left(H_{3}^{\prime \prime}\right) \quad \exists \gamma_{j}>0: \phi_{j}(y) \geq \gamma_{j}\|y\| \quad \forall y \in \mathcal{K}$

$\left(H_{8}^{\prime}\right) \quad \exists C>0: \Delta^{\star} \cap\left(-\mathcal{K}^{\star}\right) \subset B(0, C)$

$\left(H_{13}\right) \quad \exists G>0: \sum_{j=1}^{m} \phi_{j}(y) \leq G\|y\| \quad \forall y \in \mathcal{K}$.

Moreover, let one of the two assumptions below hold:

$\left(H_{10}^{\prime \prime}\right) \quad \partial f_{j}(0)=\partial f_{j}^{\star}(0)=\emptyset, \quad j=1, \ldots, m$,

or

$\left(H_{12}\right) \quad \lim \sup _{\|y\| \rightarrow \infty} \frac{\left\langle y, \partial f_{j}(y)\right\rangle}{\phi_{j}(y)}<0, \quad j=1, \ldots, m$.

Then there exist $\left(x^{\beta},\left(\pi_{j}^{\beta}\right),\left(\beta_{j}\right)\right)$ such that (92) and (93) hold.

Proof The case $\left(H_{10}^{\prime \prime}\right)$ has been already considered in Proposition 14.

Suppose that $\left(H_{12}\right)$ is satisfied. Fix any $\varepsilon \in(0,1)$ and introduce the data system on $(X \times \mathbb{R}) \times\left(X^{\star} \times \mathbb{R}\right)$ by

$$
\left.\begin{array}{l}
\widetilde{f}_{j}^{\star^{\varepsilon}}(\tau, q)=f_{j}^{\star}(\tau)+\varphi^{\varepsilon}(q), \tau \in \mathcal{K}^{\star}, q \in \mathbb{R}_{-} \\
\tilde{f}_{0}^{\varepsilon}(y, z)=f_{0}(y)+z, y \in \mathcal{K}, \quad z \in \mathbb{R}_{+} \\
\widetilde{\phi}_{j}^{\varepsilon}(y, z)=\phi_{j}(y)+s_{j} z, \quad y \in \mathcal{K}, z \in \mathbb{R}_{+},
\end{array}\right\}
$$


where $\varphi^{\varepsilon}(q)=-\varepsilon(-q)^{1-\varepsilon}$ and $s_{j}>0$ are a positive constants with $\sum_{j=1}^{m} s_{j}=1$. Note that $\partial \widetilde{f}_{j}^{\star^{\varepsilon}}(0,0)=\emptyset$ and $\partial\left(\widetilde{f}_{j}^{\star^{\varepsilon}}\right)^{\star}(0,0)=\emptyset$. The remaining assumptions of Proposition 14 hold as well. Therefore, for sufficiently large $c$ there exists $\left(x^{s}, z^{s},\left(\pi_{j}^{s}\right),\left(q_{j}^{s}\right),\left(\beta_{j}^{s}\right)\right)$ with $\left\|x^{s}\right\|+z^{s}=c^{s}$. such that

$$
\begin{aligned}
\pi_{j}^{s} \in \frac{1}{\beta_{j}^{s}} \partial f_{j}\left(x^{s}\right), \quad & z^{s}=\varepsilon(1-\varepsilon)\left(\sum_{j=1}^{m} \frac{1}{\beta_{j}^{s}}\right)^{\varepsilon}, \quad-q_{j}^{s}=\frac{1}{\beta_{j}^{s}}\left(\sum_{k=1}^{m} \frac{1}{\beta_{k}^{s}}\right)^{-1} \\
- & \sum_{j=1}^{m} \pi_{j}^{s} \in \partial f_{0}\left(x^{s}\right), \quad-\sum_{j=1}^{m} q_{j}^{s}=1 \\
& -\left\langle x^{s}, \pi_{j}^{s}\right\rangle-\phi_{j}\left(x^{s}\right)-z^{s}\left(s_{j}+q_{j}^{s}\right)=0 .
\end{aligned}
$$

From $(95)_{3}$ we get

$$
-\frac{z^{s}}{\phi_{j}\left(x^{s}\right)}\left(s_{j}+q_{j}^{s}\right) \in 1+\frac{1}{\beta_{j}^{s}} \frac{\left\langle x^{s}, \partial f_{j}\left(x^{s}\right)\right\rangle}{\phi_{j}\left(x^{s}\right)} .
$$

Taking into account $\left(H_{12}\right)$ we can assume that

$$
\frac{\left\langle x^{s}, \partial f_{j}\left(x^{s}\right)\right\rangle}{\phi_{j}\left(x^{s}\right)} \leq-\rho, \quad j=1, \ldots, m
$$

for some $\rho>0$. Thus

$$
-\frac{z^{s}}{\phi_{j}\left(x^{s}\right)}\left(s_{j}+q_{j}^{s}\right) \leq 1-\frac{\rho}{\beta_{j}^{s}}
$$

from which it follows

$$
-z^{s}\left(s_{j}+q_{j}^{s}\right) \leq \phi_{j}\left(x^{s}\right)-\frac{\rho}{\beta_{j}^{s}} \phi_{j}\left(x^{s}\right) \leq \phi_{j}\left(x^{s}\right)-\min _{j=1, \ldots, m}\left\{\gamma_{j}\right\}\left\|x^{s}\right\| \frac{\rho}{\beta_{j}^{s}} .
$$

Adding by sides these inequalities (recall that $\sum_{j=1}^{m} s_{j}=-\sum_{j=1}^{m} q_{j}=1$ ) yields

$$
0 \leq f_{0}\left(x^{s}\right)-\rho\left\|x^{s}\right\| \min _{j=1, \ldots, m}\left\{\gamma_{j}\right\} \sum_{j=1}^{m} \frac{1}{\beta_{j}^{s}} \leq G\left\|x^{s}\right\|-\rho\left\|x^{s}\right\| \min _{j=1, \ldots, m}\left\{\gamma_{j}\right\} \sum_{j=1}^{m} \frac{1}{\beta_{j}^{s}}
$$

Hence

$$
\sum_{j=1}^{m} \frac{1}{\beta_{j}^{s}} \leq \frac{G}{\rho \min _{j=1, \ldots, m}\left\{\gamma_{j}\right\}}
$$

and, consequently,

$$
-q_{j}^{s}=\frac{1}{\beta_{j}^{s}}\left(\sum_{k=1}^{m} \frac{1}{\beta_{k}^{s}}\right)^{-1} \geq \frac{\rho \min _{j=1, \ldots, m}\left\{\gamma_{j}\right\}}{S G}, \quad j=1, \ldots m .
$$


Accordingly, it has been shown that for a convex, closed set $Q$ given by

$$
Q=\left\{\left(s_{j}\right): s_{j} \geq \frac{\rho \min _{j=1, \ldots, m}\left\{\gamma_{j}\right\}}{S G}, \sum_{j=1}^{m} s_{j}=1\right\}
$$

the implication holds

$$
s=\left(s_{j}\right) \in Q \Longrightarrow-q^{s}=\left(-q_{j}^{s}\right) \in Q .
$$

Following the lines of the proof of Proposition 13 we assert that the mapping $\mathcal{S}$ which assigns to any $s \in Q$ the set of all corresponding $q^{s}$ 's, i.e. $\mathcal{S}(s)=\left\{q^{s}\right\}$, is closed and compact valued. Moreover, if $Q \ni s^{n} \rightarrow s \in Q$ as $n \rightarrow \infty$, then each cluster point of $\left\{q^{s^{n}}\right\}$ lies in $\mathcal{S}(s)$. Again, by the Ky Fan inequality the existence of a fixed point of $\mathcal{S}$ is ensured. By $(95)_{3}$ we arrive at the desired conclusion. The proof is complete.

Acknowledgement The author is very thankful to the reviewer for his valuable remarks which essentially improved the final version of this paper.

Open Access This article is distributed under the terms of the Creative Commons Attribution Noncommercial License which permits any noncommercial use, distribution, and reproduction in any medium, provided the original author(s) and source are credited.

\section{References}

1. Aliprantis, C.D., Brown, D.J., Burkinshaw, O.: Existence and Optimality of Competitive Equilibria. Springer, Berlin (1989)

2. Aliprantis, C.D., Florenzano, M., Tourky, R.: Linear and non-linear price decentralization. J. Econ. Theory 121, 51-74 (2005)

3. Aliprantis, C.D., Monteriro, P.K., Tourky, R.: Non-marketed options, non-existence of equilibria, and nonlinear prices. J. Econ. Theory 114, 345-357 (2004)

4. Aliprantis, C.D., Tourky, R., Yannelis, N.C.: A theory of value with nonlinear prices. J. Econ. Theory 100, 22-72 (2001)

5. Arrow, K., Debreu, G.: Existence of an equilibrium for a competitive economy. Econometrica 22, 264-290 (1954)

6. Arrow, K.J., Intrilligator, M.D.: Handbook of Mathematical Economics, vol. II. North-Holland, Amsterdam (1982)

7. Attouch, H., Brézis, H.: Duality for the sum of convex functions. In: Barroso, J.A. (ed.) Aspects of Mathematics and its Applications. Elsevier, Amsterdam (1986)

8. Aubin, J.P.: Optima and Equilibria. Springer, Berlin (1993)

9. Baiocchi, C., Buttazzo, G., Gastaldi, F., Tomarelli, F.: General existence theorems forunilateral problems in continuum mechanics. Arch. Ration. Mech. Anal. 100, 149-188 (1988)

10. Bao, T.Q., Gupta, P., Mordukhovich, B.S.: Necessary conditions in multiobjective optimization with equilibrium constraints. J. Optim. Theory Appl. 134, 179-203 (2007)

11. Breckner, W.W., Kolumbán, I.: Dualität bei optimierungsaufgaben in topologischen vektorräumen. Mathematica 10(33), 229-244 (1968)

12. Browder, F.E., Hess, P.: Nonlinear mappings of monotone type in Banach spaces. J. Funct. Anal. 11, 251-294 (1972)

13. Chen, G.Y., Huang, X.X., Yang, X.Q.: Vector optimization: set-valued and variational analysis. In: Lect. Notes Econ. Math. Syst., vol. 541. Springer (2005)

14. Chichilnisky, G.: A unified perspective on resource allocation: limited arbitrage is necessary and sufficient for the existence of a competitive equilibrium, the core and social choice. In: Chichilnisky, G. (ed.) Topology and Markets. American Mathematical Society and the Fields Institute for Research in Mathematical Sciences, Toronto (1999) 
15. Chichilnisky, G., Heal, G.: A unified treatment of finite and infinite economies: limited arbitrage is necessary and sufficient for the existence of equilibrium and the core. Econ. Theory 12, 163-176 (1998)

16. Eaves, C.: Homotopies for computation of fixed points. Math. Program. 3,1-22 (1972)

17. Ekeland, I., Temam, R.: Convex Analysis and Variational Problems. North-Holland, Amsterdam (1976)

18. Elster, R., Gerth, C., Göpfert, A.: Duality in geometric vector optimization. Optimization 20, 457-476 (1989)

19. Gale, D., Mas-Colell, A.: An equilibrium existence theorem for a general model without ordered preferences. J. Math. Econo. 2, 9-15 (1975)

20. Geoffrion, A.M.: Proper efficiency and the theory of vector maximization. J. Math. Anal. Appl. 22, 618-630 (1968)

21. Göpfert, A., Riahi, H., Tammer, C., Zalinescu, C.: Variational methods in partially ordered spaces. In: CMS Books in Mathematics, vol. 17. Springer (2003)

22. Hirsh, M., Smale, S.: On algorithms for solving $f(x)=0$. Commun. Pure Appl. Math. 32, 281-312 (1979)

23. Jahn, J.: Vector Optimization: Theory, Applications and Extensions. Series Oper. Res. Springer (2004)

24. Jofré, A., Rockafellar, R.T., Wets, R.J-B.: Variational inequalities and economic equilibrium. Math. Oper. Res. 32, 32-50 (2007)

25. Luc, D.T.: Theory of vector optimization. In: Lecture Notes in Economics and Mathematical Systems, vol. 139. Springer (1989)

26. Mas-Colell, A.: The price equilibrium existence problem in topological vector lattices. Econometrica 54, 1039-1053 (1986)

27. Mas-Colell, A., Richard, S.F.: A new approach to the existence of equilibria in vector lattices. J. Econ. Theory 53,1-11 (1991)

28. Mas-Colell, A., Whinston, M.D., Green, J.R.: Microeconomic Theory. Oxford University Press, New York (1995)

29. McKenzie, L.: On the existence of general equilibrium for a competitive market. Econometrica 27, 54-71 (1959)

30. Miettinen, K.M.: Nonlinear Multiobjective Optimization. Kluwer, Boston (1998)

31. Mordukhovich, B.S.: Variational analysis and generalized differentiation. I: Basic theory. In: Grundlehren Series (Fundamental Principles of Mathematical Sciences), vol. 330. Springer, Berlin (2006)

32. Mordukhovich, B.S.: Variational analysis and generalized differentiation. II: Applications. In: Grundlehren Series (Fundamental Principles of Mathematical Sciences), vol. 331. Springer, Berlin (2006)

33. Nagurney, A., Siokos, S.: Financial Networks: Statics and Dynamics. Springer, Berlin (1997)

34. Naniewicz, Z.: Pseudo-monotonicity and economic equilibrium problem in reflexive Banach space. Math. Oper. Res. 32, 436-466 (2007)

35. Naniewicz, Z.: Pareto optimality and Walrasian equilibria. J. Math. Anal. Appl. 341, 1365-1381 (2008)

36. Nash, J.F.: Equilibrium points in n-person games. Proc. Natl. Acad. Sci. U. S. A. 36, 48-49 (1950)

37. Negishi, T.: Welfare economics and existence of an equilibrium for a competitive economy. Metroeconomica 12, 92-97 (1960)

38. Neumann, V.J.: A model of general economic equilibrium. Rev. Econ. Stud. XIII, 1-9 (19451946). G. Morgenstern, transl.

39. Nockowska-Rosiak, M.: Economic equilibrium through variational inequalities. Appl. Math. 36, 43-58 (2009)

40. Pallaschke, D., Rolewicz, S.: Foundation of Mathematical Optimization. Kluwer, Boston (1997)

41. Peressini, A.L.: Ordered topological vector spaces. Harper \& Row, New York (1967)

42. Rockafellar, R.T.: Extension of Fenchel's duality theorem for convex functions. Duke Math. J. 33, 81-90 (1966)

43. Rockafellar, R.T.: Convex Analysis. Princeton University Press, Princeton (1970)

44. Sawaragi, Y., Nakayama, H., Tanino, T.: Theory of Multiobjective Optimization. Academic, New York (1985)

45. Scarf, H.E.: The Computation of Economic Equilibria. Yale University Press, New Haven (1973)

46. Smale, S.: Exchange processes with price adjustment. J. Math. Econ. 3, 211-226 (1976)

47. Tanino, T.: Conjugate duality in vector optimization. J. Math. Anal. Appl. 167, 84-97 (1992) 
48. Wanka, G., Boţ, R.-I.: Multiobjective duality for convex-linear problems II. Math. Methods Oper. Res. 53, 419-433 (2001)

49. Wanka, G., Bot, R.-I.: A new duality approach for multiobjective convex optimization problems. J. Nonlinear Convex Anal. 3, 41-57 (2002)

50. Wanka, G., Boţ, R.-I.: Duality for multiobjective optimization problems with convex objective functions and D.C. constraints. J. Math. Anal. Appl. 315, 526-543 (2006) 